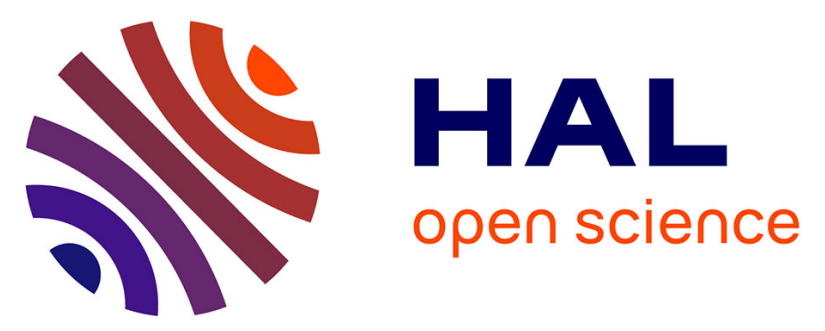

\title{
Investigation of adaptive optics performance through propagation channel characterization with the Small Optical TrAnsponder SOTA
}

\author{
Cyril Petit, Nicolas Védrenne, Marie-Thérèse Velluet, Vincent Michau,
} Géraldine Artaud, Etienne Samain, Morio Toyoshima

\section{To cite this version:}

Cyril Petit, Nicolas Védrenne, Marie-Thérèse Velluet, Vincent Michau, Géraldine Artaud, et al.. Investigation of adaptive optics performance through propagation channel characterization with the Small Optical TrAnsponder SOTA. Optical Engineering, 2016, 55 (11), pp.111611. 10.1117/1.OE.55.11.111611 . hal-01398000

\section{HAL Id: hal-01398000 https://hal.science/hal-01398000}

Submitted on 16 Nov 2016

HAL is a multi-disciplinary open access archive for the deposit and dissemination of scientific research documents, whether they are published or not. The documents may come from teaching and research institutions in France or abroad, or from public or private research centers.
L'archive ouverte pluridisciplinaire HAL, est destinée au dépôt et à la diffusion de documents scientifiques de niveau recherche, publiés ou non, émanant des établissements d'enseignement et de recherche français ou étrangers, des laboratoires publics ou privés. 


\title{
Investigation of adaptive optics performance through propagation channel characterization with the Small Optical TrAnsponder SOTA
}

\author{
C. Petit, ${ }^{* a}$ N. Védrenne, ${ }^{a}$ M.-T. Velluet, ${ }^{a}$ V. Michau, ${ }^{a}$ G. Artaud, ${ }^{b}$ E. Samain, ${ }^{c}$ M. \\ Toyoshima, ${ }^{\mathrm{d}}$
}

${ }^{a}$ ONERA, The French Aerospace Lab, Theoretical and Applied Optics Department, 92322 Châtillon Cedex, France

${ }^{\mathrm{b}}$ CNES, DCT/RF/ITP, 18, av. Edouard Belin, 31401 Toulouse Cedex 9, France

${ }^{\mathrm{c}}$ OCA-Géoazur, 2130, Route de l'Observatoire, 06460 Saint-Vallier-de-Thiey, France

${ }^{\mathrm{d}}$ National Institute of Information and Communications Technology, Space Communication Systems Laboratory, 4-

2-1 Nukui-Kitamachi, Koganei, Tokyo 184-8795 Japan

\begin{abstract}
In order to address the high throughput requested for both downlink and uplink satellite to ground laser links, Adaptive Optics (AO) has become a key technology. While mature, application of this technology to satellite to ground telecommunication faces however difficulties such as higher bandwidth, optimal operation for a wide variety of atmospheric conditions (daytime and nighttime) with potentially low elevations that might severely affect wavefront sensing because of scintillation. To address these specificities an accurate understanding of the origin of the perturbations is required, as well as operational validation of $\mathrm{AO}$ on real laser links. We report here on a Low Earth Orbiting (LEO) microsatellite to ground downlink with AO correction. We discuss propagation channel characterization based on Shack-Hartmann WaveFront Sensor (WFS) measurements. Fine modelling of the propagation channel is proposed based on multi-Gaussian model of turbulence profile. This model is then used to estimate the AO performance and validate the experimental results. While AO performance is limited by the experimental set-up, it proves to comply with expected performance and further interesting information on propagation channel is extracted. These results shall help dimensioning and operating AO systems for LEO to ground downlinks.
\end{abstract}

Keywords: Adaptive optics, optical turbulence, free space laser communications, wavefront sensing, scintillation

*Cyril Petit, E-mail: cyril.petit@onera.fr

\section{Introduction}

High data rate optical communications from satellite (Low Earth Orbit -LEO- or Geostationary

Earth Orbit -GEO- ) to ground have been under investigation for several years; they offer a good alternative to the Radio Frequency ( $R F$ ) links which are approaching the limit of their capabilities in terms of data throughput. However, one of the major issues is the atmospheric turbulence which disrupts the propagation channel. Both phase and amplitude of the optical wave are perturbed leading to power fluctuations of the signal and potential deep fading. Mitigation techniques can be applied on the signal itself (interleaving or corrector error codes) and/or on the 
wavefront thanks to Adaptive Optics (AO). To address very high data rate at affordable cost, the use of single mode components is necessary. Turbulence influence can significantly degrade the optical quality of the incoming wave, thus severely degrading the efficiency of the wave coupling into the fiber. The real time compensation of phase disruptions by AO can solve this issue. However, LEO satellite Direct To Earth (DTE) optical links represent a particularly challenging framework for $\mathrm{AO}$ due to harsh conditions, involving scintillation, fast evolving turbulence and strong operational constraints. Although various AO systems dedicated to ground-to-space communications have been proposed or tested in lab ${ }^{1,2,3}$, until recently no demonstration of ground-based AO for optical downlink was reported. In 2015 NASA demonstrated optical link with International Space Station using the Optical PAyload for Lasercomm Science (OPALS) and AO for a 134 seconds connection, paving the way to AO for downlinks ${ }^{4}$. AO demonstration for coherent detection was also presented by Tesat in October $2015^{5}$. However, a lot needs to be done to understand performance limitations of the reported results, particularly concerning the influence of wavefront residuals on the coupling efficiency. Thus, no direct link has been performed up to now between turbulent conditions and AO performance obtained during experiment. In this paper we report the results of AO correction performed with the $976 \mathrm{~nm}$ laser link provided by the Small Optical TrAnsponder (SOTA) terminal embedded on the Space Optical Communications Research Advanced Technology Satellite (SOCRATES). These results are compared to a simplified simulation tool, presented in $^{6,7}$.

First, the propagation channel characterization is performed, prior to AO performance analysis. It brings critical information about turbulence statistics to be considered for LEO to ground optical links and AO system dimensioning and operating. These statistics are then used to define the 
simulation conditions. Turbulence conditions parametrization $\left(\mathrm{C}_{\mathrm{n}}{ }^{2}\right.$ profile $)$ of the simulation is derived from the data recorded by the wavefront sensor. Finally, the simulation tool allows an fine estimation of $\mathrm{AO}$ performance that can be compared with the actual experimental $\mathrm{AO}$ performance. An accurate understanding of the global AO performance including the coupling efficiency is thus obtained based on the exhaustive analysis of the propagation channel. This approach shall provide significant clues for AO system design and dimensioning for LEO to ground downlinks but also uplinks and endo-atmospheric communication.

The first part of the paper presents the experimental setup and the collected data. The estimation of the turbulence conditions along the link is presented in the second part. The third part is dedicated to the comparison of $\mathrm{AO}$ results with a numerical model.

\section{Experimental Set-up}

\subsection{The Demonstrator for direct Optical transMission at hIgh data rate iN Orbit (DOMINO)} project

The data collection presented here was conducted in July 2015 in the framework of a cooperation agreement on information and communication technology signed between National Institute of Information and Communications Technology (NICT) and Centre National d'Etudes Spatiales (CNES). In this context the $\mathrm{MeO}$ telescope (for "Metrology and Optics") of Observatoire de la Côte d'Azur (OCA) has been adapted to serve as a ground station for the laser link. The adaptation and the data acquisition were performed within the DOMINO project. CNES is the project owner and OCA the leader. ONERA was in charge of turbulence characterization and adaptive optics, Airbus Defense of system analysis and Thales Alenia Space of communication aspects. 


\subsection{MeO ground station}

The $\mathrm{MeO}$ station is a versatile telescope located in the hinterland of Grasse at an altitude of 1270 meters, up to now mostly used for artificial satellites and lunar laser ranging ${ }^{8}$. This 1.5 meter telescope (Fig. 1) is installed on an Alt-Az mount allowing target tracking up to $5 \%$ s, with a 20" Peak-Valley (PV) pointing accuracy thanks to a dedicated tracking loop. These features make this telescope a very good candidate for ground to LEO satellite communication demonstration. The station participates also to several research programs such as optical time transfer, adaptive optics, high resolution imagery, debris detection, optical telecom and astronomical observation.

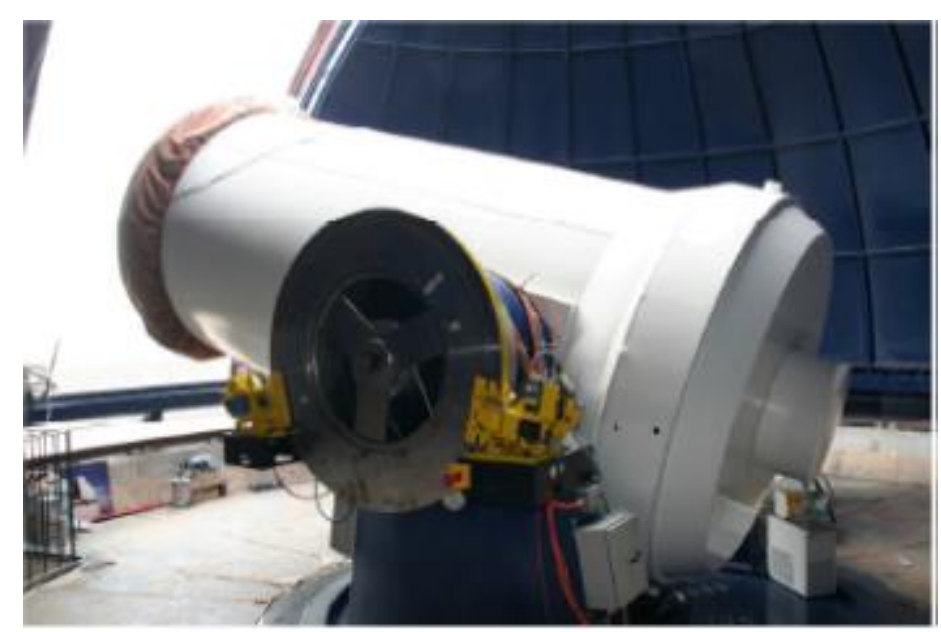

Fig. 1 Picture of $\mathrm{MeO}$ telescope.

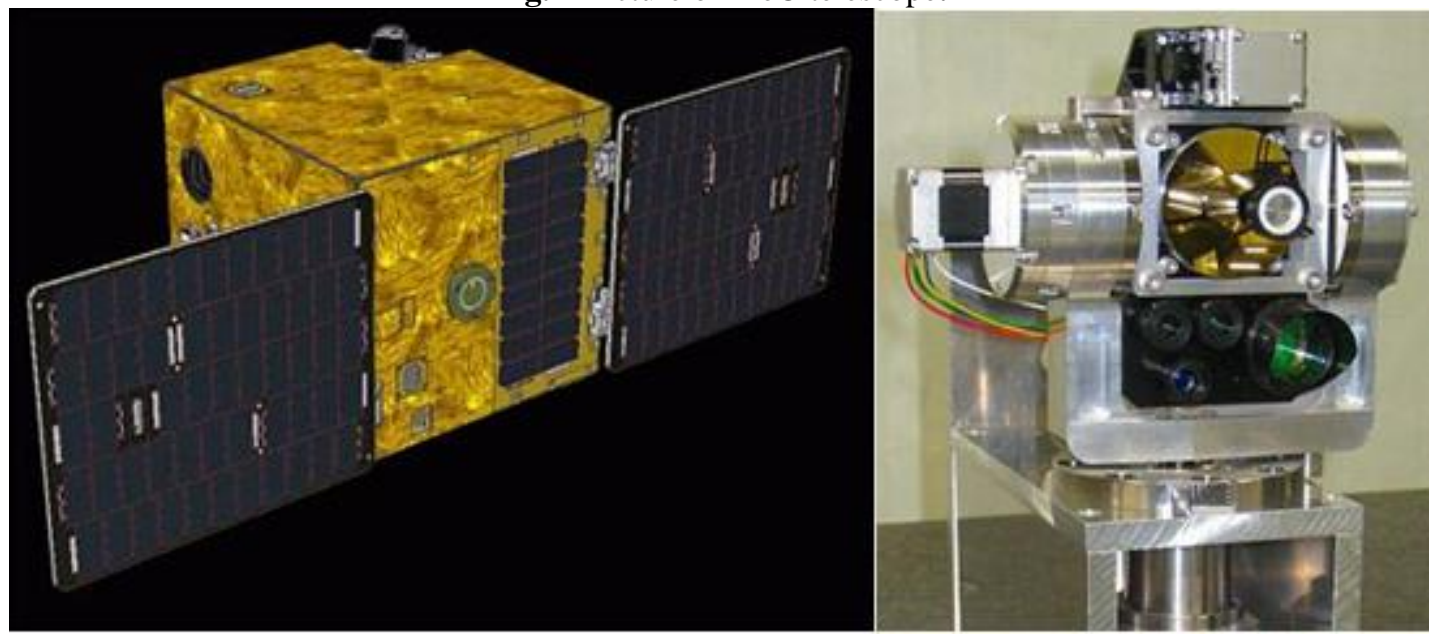

Fig. 2 Illustration of SOCRATES (credit AES) (left) and picture of SOTA (credit NICT, NEC) (right). 


\subsection{SOCRATES micro satellite}

SOCRATES (Fig. 2 left) is a microsatellite mission carrying SOTA, a technology demonstrator for laser communications with micro-satellites developed by NICT $^{9}$ (Fig. 2 right). The light emitted by the transponder is collected on ground by MeO. SOTA provides two possible downlink wavelengths: $1549 \mathrm{~nm}$ and $976 \mathrm{~nm}$. The future optical space transmission operational systems in which CNES might be involved will use $1.55 \mu \mathrm{m}$ wavelength range for uplink and downlink, according to aimed future standards under development (see Consultative Committtee

for Space Data Systems ${ }^{10}$ for instance), compatible with the LEO DTE transmission scenario. For this experiment, the $976 \mathrm{~nm}$ modulated laser with $200 \mathrm{~mW}$ average power was used in order to benefit from the existing ODISSEE (Observation, Déconvolution et Imagerie depuis le Sol de Satellites et de l'EspacE) optical bench available at OCA. The alignment of SOTA terminal with $\mathrm{MeO}$ ground station is performed thanks to a laser beacon installed on $\mathrm{MeO}$. This operation is performed when SOTA is not sun lightened. During the experiment ODISSEE bench has been used to characterize turbulence influence on the propagation channel and perform $\mathrm{AO}$ correction. Simultaneously, Non Return to Zero-On-Off Keying (NRZ OOK) power modulations for a 10 Mbps transmission were recorded with a dedicated instrument located at the Nasmyth focus of the telescope. Data transmission results are presented elsewhere ${ }^{11}$.

\subsection{ODISSEE AO bench}

The ODISSEE AO bench has been designed and integrated by ONERA at the Coudé focus of $\mathrm{MeO}^{7}$ for investigation of various applications, including visible imaging. In this prospect, it has been adapted from an existing laboratory $\mathrm{AO}$ bench, favoring versatility rather than performance. As a consequence, this $\mathrm{AO}$ system is neither designed for telecommunication application, as we shall underline later on, nor for the typical turbulent conditions encountered at OCA. However it 
provides a useful tool to assess propagation conditions and performance of new concepts, and investigate the limitations of $\mathrm{AO}$ for this application. The decision was therefore taken to opportunely close the loop on SOTA laser signal using this system. Fig. 3 provides a schematic description of the bench.

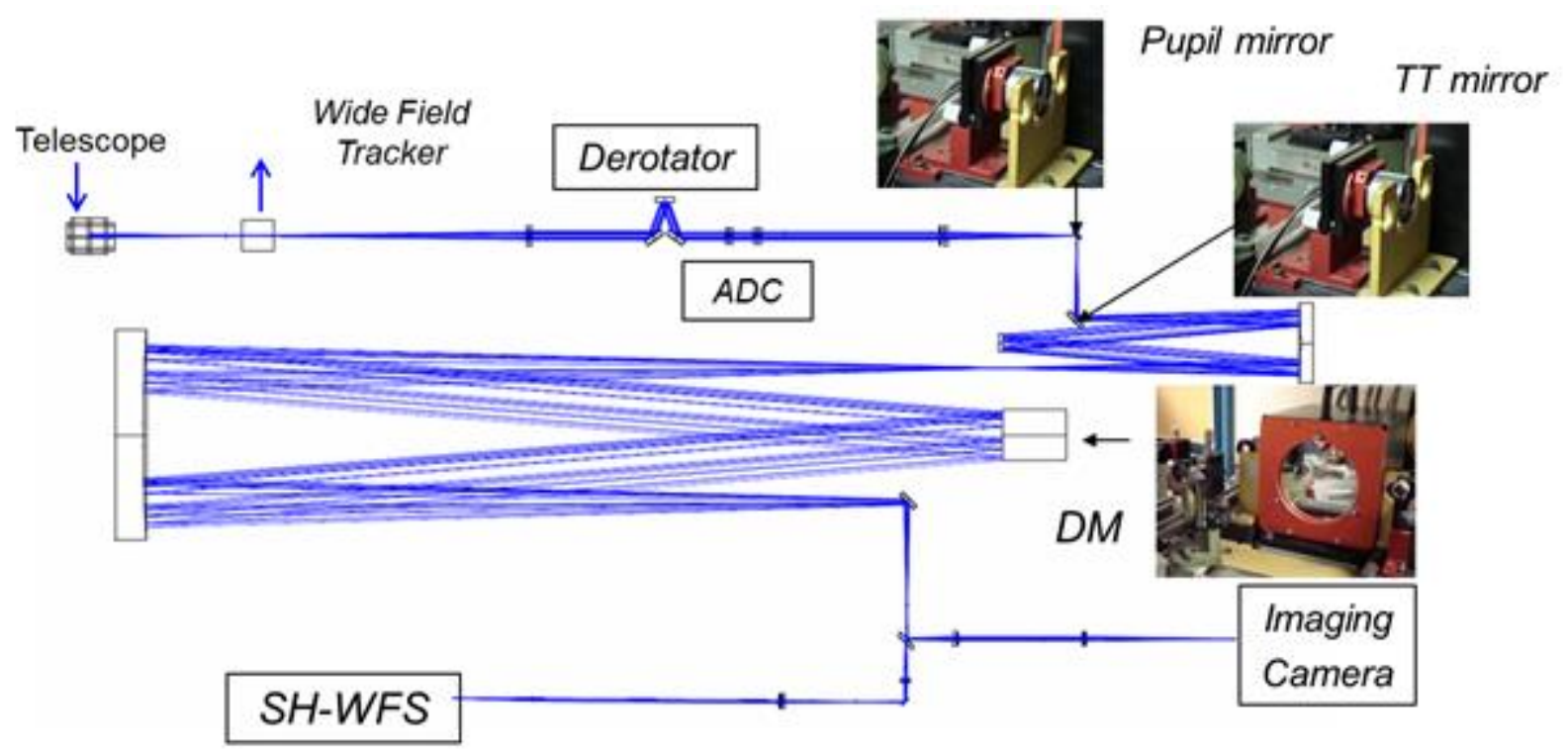

Fig. 3 Schematic of the ODISSE AO bench at MeO Coudé focus.

The light from Coudé focus enters the bench at the top-left of the image. The pupil derotator compensates for the field rotation. The pupil position is stabilized with a slow tip/tilt mirror located close to a focal plane. A second fast tip/tilt mirror is used to compensate for atmospheric turbulent tip/tilt, pointing residuals and vibrations. After reflecting on the deformable mirror, the beam is split and directed onto the WFS and the imaging camera.

The point spread function of the corrected beam is observed on an imaging camera, a PCO Edge 5.5 scientific Complementary Metal-Oxide-Semiconductor (sCMOS) detector used on a $480 \times 480$ pixel region $(6.5 \mu \mathrm{m}$ pixel size). This scientific path is usually dedicated to imaging at Shannon sampling at $850 \mathrm{~nm}$. In this configuration, the output numerical aperture is thus not adapted to coupling the signal into single mode fibers. 
The AO bench also implements an Atmospheric Dispersion Compensator (ADC) but as the derotator it is not used in this experiment.

\subsubsection{Wavefront sensor of ODISSEE}

ODISSEE uses a Shack-Hartmann WaveFront Sensor (SH-WFS) with 8x8 square sub-apertures. The field of each sub-aperture covers 30x30 pixels in the focal plane. The focal length of the micro-lenses has been chosen so that sampling factor is 0.5 Shannon at $600 \mathrm{~nm}$ (one diffraction spot per pixel). The WFS camera is a First Light Imaging OCAM2 camera (Electron Multiplying Charge Coupled Device -EMCCD-) with an engineering grade E2V chip of 240x240 pixels providing 0.3 quantum efficiency at $970 \mathrm{~nm}$. Slopes and intensities per subaperture are recorded with a frame rate of $1450 \mathrm{~Hz}$. The slope computation algorithm is a thresholded Center of Gravity (CoG). The threshold value is adjusted to limit background noise influence on the slope measurements. The total intensity in each sub-aperture is computed by the summation of the pixel thresholded values.

\subsubsection{Deformable mirror of ODISSEE}

High order correction is performed thanks to a CILAS piezo-stacked Deformable Mirror (DM) with 88 actuators (10x10 cartesian grid), though the useful aperture covers only a 9x9 actuator area of the DM (see Fig. 4). All actuators are used for real-time correction. It provides a $+/-5 \mu \mathrm{m}$ mechanical stroke and exhibits a $10 \mathrm{kHz}$ bandwidth. 


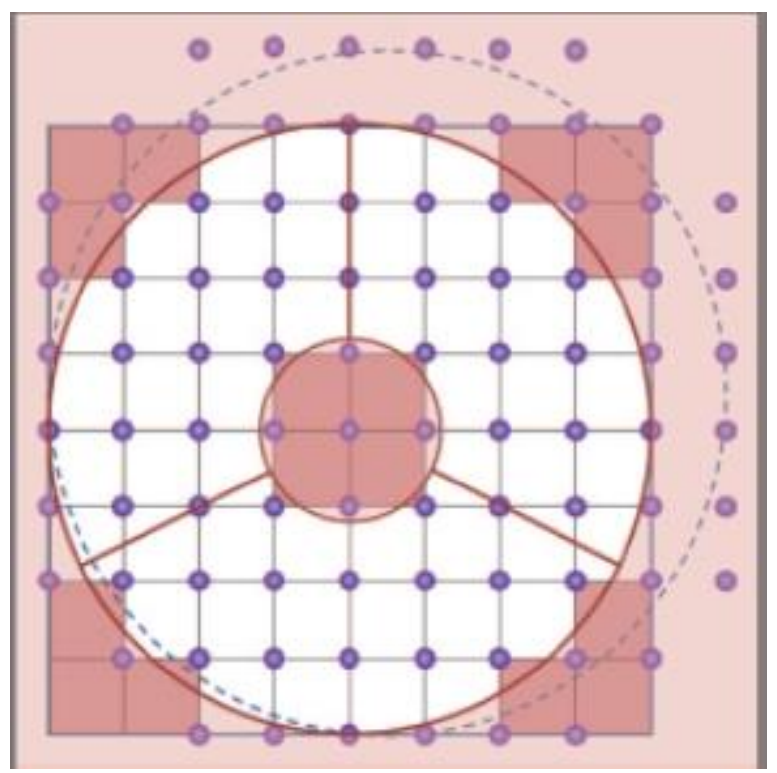

Fig. 4 Schematic of the DM actuator grid (dots), located at the angles of SH-WFS subaperture grid (squares). The useful aperture, with telescope spider and central occultation are delimited in red. A 8x8 sub aperture grid is used on SH-WFS, valid subapertures are whitened.

\subsubsection{Real Time Computer}

The AO loop is controlled thanks to a Linux Personal Computer (PC) Real-Time Controller (RTC) implementing various possible features both in terms of wavefront sensing or control algorithms. Still, for this particular application, a high sampling frequency is targeted. Consequently, the simplest configuration of RTC is used to reach a $1.45 \mathrm{kHz}$ sampling frequency. In these conditions, the overall loop delay is 3.3 frames, due to RTC latency, highlighting the inadequacy of the RTC to manage such high temporal frequency application. Thus, wavefront sensing is performed using simple thresholded CoG, without particular handling of possible scintillation, and a standard integrator based control loop, with fixed scalar gain, so as to reduce computational burden and latency. Clearly, much gain in performance could be expected by optimization of RTC (hardware and software) to reduce latency and increase the effective $0 \mathrm{~dB}$ bandwidth (currently limited to $55 \mathrm{~Hz}$ ). Application of more refined control laws, at least optimized modal gain integrator, or optimal predictive control (linear quadratic Gaussian 
approach) would be also of interest considering the impact of temporal error and the presence of vibrations as discussed later ${ }^{12}$. Pupil stabilization is performed at slow rate $(10 \mathrm{~Hz})$ based on a simple integrator feedback loop.

The internal performance of the AO bench in this configuration (defined as Strehl Ratio of a non-resolved source at entrance focus) is $91 \%$ at $976 \mathrm{~nm}$ (representing the ultimate performance of the system on sky), due to residual aberrations (high order residuals and non-common path aberrations).

\subsection{Experiment and data acquired}

The space-to-ground experiment has been carried out on the 21st of July 2015 Coordinated Universal Time (UTC) by night. Only one run was performed due to weather conditions and SOTA terminal availability, leading to limited but fruitful data collection. SOCRATES satellite was visible from $22: 34: 13 \mathrm{~s}$ UTC ( $4^{\circ}$ elevation) to $22: 38: 41 \mathrm{~s}\left(44.5^{\circ}\right.$ elevation, SOTA terminal being shut down afterwards - see Fig. 5), during which we performed first an AO free analysis of the incoming laser beam $\left(7.5^{\circ}\right.$ to $21^{\circ}$ elevation), thanks to the SH-WFS, both for turbulent beam characterization and WF Sensing optimization. Then AO loop was optimized and closed on the downlink laser beam. A stable and continuous AO correction was provided over 145 s. Of course, closed-loop data benefit from slightly better turbulent conditions as loop is closed for higher elevations. Thus $r_{0}$ at $\lambda=500 \mathrm{~nm}$ goes from $4.5 \mathrm{~cm}$ in open-loop phase to $6 \mathrm{~cm}$ in closed loop phase. In the meantime, temporal error may be increased due to higher satellite velocity (see Fig. 5) leading to increased apparent wind velocity (Greenwood frequency dependency with respect to wind and slew rate for instance is discussed $\mathrm{in}^{13}$ ). 
During each phase (open then closed loop, see Fig. 5), both real-time data (SH-WFS slopes and intensities and DM controls) and images have been acquired. Sequences of 256 frames were acquired on the PCO detector during each phase, with a $100 \mathrm{~ms}$ exposure time in open-loop and $10 \mathrm{~ms}$ in closed-loop (period of acquisition identified in Fig. 5), due to concentration of energy. Number of sequences acquisition is strongly limited by the time of transfer of the PCO camera. The WFS data were collected. From the beginning to the end of the acquisition, $152 \mathrm{~s}$ of slopes and intensities were recorded with a framerate of $1450 \mathrm{~Hz}$. At 22:34:58, 200 raw images from the WFS were acquired (elevation $7.5^{\circ}$ ). Between 22:35:40 and 22:35:55 the parameters of the WFS (camera gain, threshold level, and background) were adjusted. Data acquisition (slopes and intensities) was launched at 22:36:09 (elevation $13^{\circ}$ ). At 22:36:56 (elevation $21^{\circ}$ ) AO loop was closed and remained closed until 22:38:41 $\left(44.5^{\circ}\right)$. Between 22:36:09 and 22:37:30, a few parameters like the gain of the camera and the threshold level were adjusted. Pupil stabilization was also launched during this period. As a result, measurements of flux fluctuation (scintillation index) are altered during this part of the pass. The phase measurements are also disrupted by the closing of the loop and resumed when the loop is stabilized. Though the received power was modulated for the transmission of a Pseudo Random Binary Sequence of $2^{15}$ bits at10 Mbps, we did not notice any significant influence of the power modulation neither on intensities recording nor on slopes measurements as the exposure time of the WFS was several hundred times longer than the bit duration. 

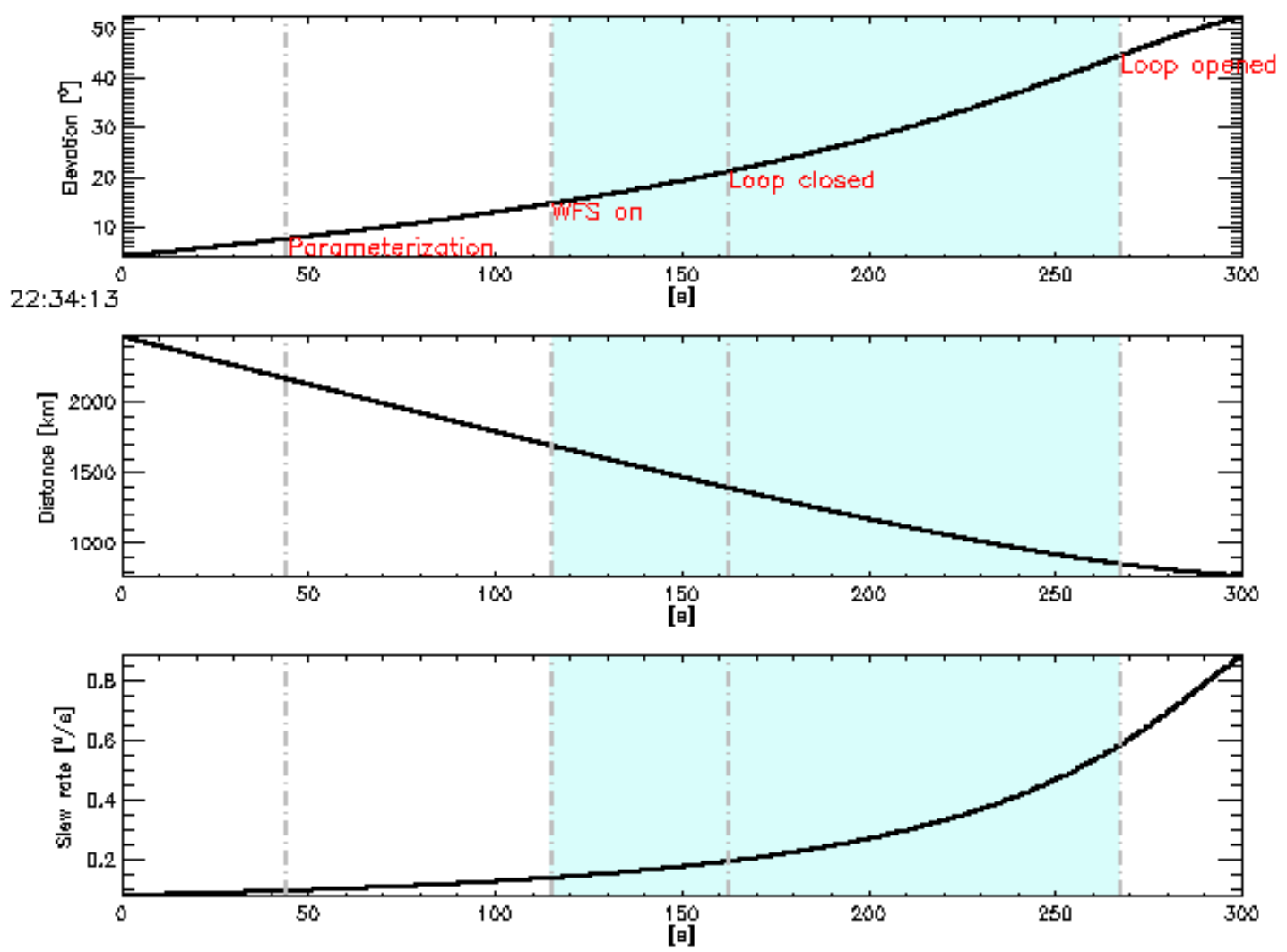

Fig. 5 Top: elevation as a function of time since the rise of the satellite. Middle: distance to satellite, bottom: antenna slew rate. Blue: WFS data acquisition.

\section{Turbulence characterization}

\subsection{Fried parameter estimation}

The wavefront is spatially sampled by the WFS. The local slope in front of each subaperture is estimated from the position of the focal spot using a thresholded CoG. The wavefront on the telescope aperture is then reconstructed by inverting a slope measurement model with a linear approach. When the AO loop is closed, pseudo-open loop slopes are computed from residual slopes and command applied to the DM.

The set of data is decomposed into 35 data subsets evenly spaced with a period of $4 \mathrm{~s}$. Each set represents a $13.8 \mathrm{~s}$ duration. For each frame, the estimated wavefront is decomposed onto the first 45 Zernike modes (piston mode excluded). For each data subset, the variances per radial 
order are computed. They are reported in Fig. 6 (squares) for five elevations $\left(15.5^{\circ}, 19.8^{\circ}, 28.2^{\circ}\right.$, $32.6^{\circ}$ and $41.9^{\circ}$ ).
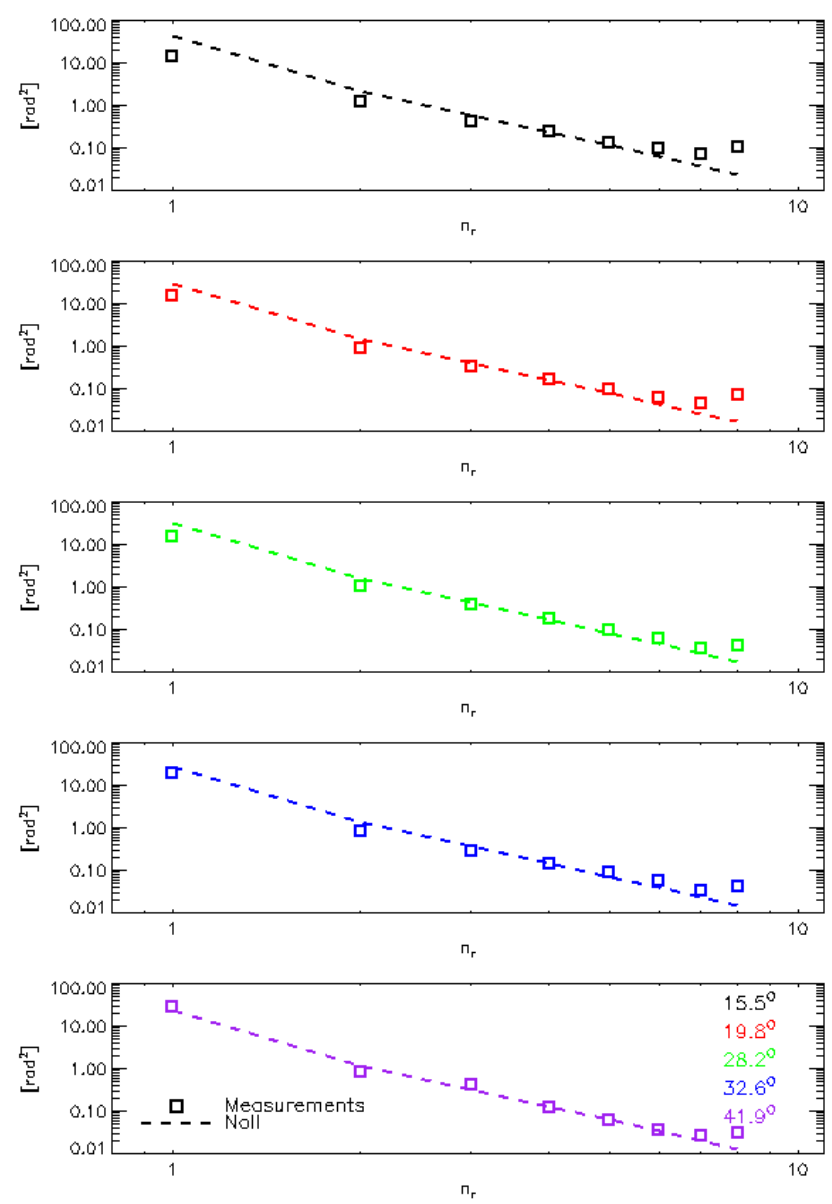

Fig. 6 Wavefront variance per Zernike polynomial radial order for the five selected elevations experimental (squares), compared to theory (- - ).

An evaluation of the Fried parameter at $\lambda=500 \mathrm{~nm}$ is performed from the radial variances given by $\mathrm{Noll}^{14}$ (see Fig. 7). The radial orders 1 and 2 are not used in the $r_{0}$ estimation because they integrate the effect of the turbulence but also the contribution of vibrations, pointing error and outer scale, which could compromise the reliability of $\mathrm{r}_{0}$ estimation.

To avoid the influence of aliasing, only radial orders up to six are considered, therefore $\mathrm{r}_{0}$ estimation is performed for each radial order between 3 and 6 . With the average estimated $\mathrm{r}_{0}$ and 
by assuming a $-11 / 3$ power law, Zernike variances are computed (dashed lines) and compared to the measured ones (squares). For radial orders greater than 2, the $-11 / 3$ power law is well satisfied. The standard deviation on the $\mathrm{r}_{0}$ estimation is also reported in Fig. 7. It corresponds to the dispersion of the $r_{0}$, estimated from each radial order (i.e. from 3 to 6 ).

Assuming the $\mathrm{C}_{\mathrm{n}}^{2}$ vertical profile as unchanged in the surroundings of the telescope, the variation of $r_{0}$ as a function of elevation is given by ${ }^{15}$ :

$$
\mathrm{r}_{0}(\theta)=(\sin \theta)^{3 / 5} \mathbf{r}_{0},
$$

where $r_{0}$ is the Fried parameter at Zenith and $\theta$ is the elevation angle.

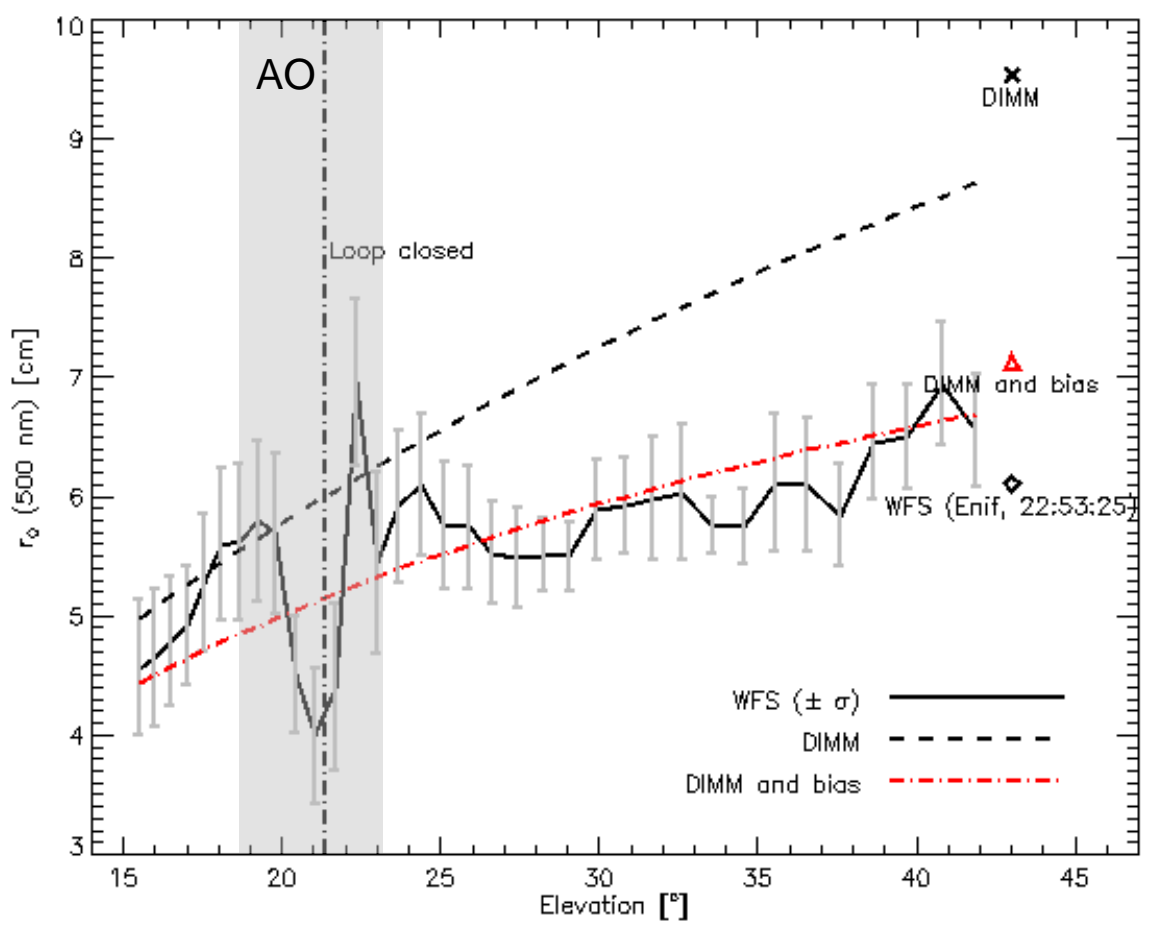

Fig. 7 Fried parameter evaluation as a function of elevation, during the pass (lines) and on a star (symbols) after the pass. Solid line corresponds to WFS measurements, each value is provided with a one sigma error bar. Dashed line corresponds to evolution as deduced from DIMM measurement and Eq. (1). Dashed dotted red line is the same as previous line with introduction of bias. Grey vertical band indicates period of time dedicated to parameters adjustment and loop closure, leading to hampered evaluation. The results of estimation from WFS data acquired on a star (Enif) at 22:53:25 is plotted in black diamonds. Simultaneous GDIMM measurement is plotted with the black cross, red triangle corresponds to the DIMM measurements with the bias. 
In parallel with the WFS acquisitions, $\mathrm{r}_{0}$ measurements are provided every 105 seconds by a Generalized Differential Image Motion Monitor (GDIMM) located several dozen meters from the telescope ${ }^{16}$. The source used for the measurements is Vega (HIP91262). Its elevation was $84^{\circ}$ at 22:00:00. The GDIMM measurement is $\mathrm{r}_{0}=11 \mathrm{~cm}$ (at zenith and at 500 $\mathrm{nm}$ ) for the time period corresponding to the wavefront sensor acquisitions. It is reported on the plot assuming that the elevation dependence obeys Eq. (1). As illustrated by the plot in Fig. 7, the value of $r_{0}(\theta)$ estimated with the beam coming from the satellite Line-Of-Sight (LOS) does not correspond to the value of $r_{0}$ obtained from GDIMM measurements. Their origin may be due, for instance, to local turbulence effects related to the $1.5 \mathrm{~m}$ telescope itself (dome effect or temperature gradients close to the tube). In this case, the biased $r_{0}$ denoted $r_{0, \text { bias }}$ depends on the elevation as:

$$
\mathrm{r}_{0, \mathrm{bias}}(\theta)=\left(\operatorname{bias}^{-5 / 3}+\mathrm{r}_{0}(\theta)^{-5 / 3}\right)^{-3 / 5}
$$

Based on this analysis, we estimate a mean value for the bias equal to $12,6 \mathrm{~cm}$. This result is in good agreement with previous analysis performed in ${ }^{17}$ in the framework of satellite imaging, in similar conditions, on stars (same telescope, same AO bench and measurement process, same cross-check with GDIMM data). This analysis had identified an artificial contribution to $\mathrm{r}_{0}$ between 8 and $12 \mathrm{~cm}$ (at 500nm) attributed to local turbulence in the dome. Once this bias is taken into account, GDIMM measurements and WFS measurements match fairly well, except while the AO loop is stabilizing. At this moment, the modifications of system parameters degrade the WFS data and may explain the important variations of Fried parameters between $19^{\circ}$ and $23^{\circ}$. Further investigations are needed to assess the reliability of the estimation in this elevation range. Outside this time window, $r_{0}$ measurements from WFS and GDIMM are consistent even if the distance between both instruments is $80 \mathrm{~m}$ and that both measurements are 
not perfectly simultaneous (the correlation time of $r_{0}$ measurements is estimated to 10 min from GDIMM measurements, which guarantees an acceptable correlation between both measurements). Note that the wind at ground level was null according to the meteorological station of the observatory. In order to cross-calibrate the $r_{0}$ estimation from WFS measurements with GDIMM measurements, $\mathrm{r}_{0}$ has also been estimated from measurements on a star (Enif, HIP107315) few minutes after the pass (at 22:53:25). The result of the measurement is reported in Fig. 7. The $r_{0}$ measurement provided by DIMM the same minute, extrapolated to the elevation of Enif is plotted with a cross. The DIMM measurement with bias is plotted with a red triangle. The proximity of both estimations tends to consolidate the hypothesis of a bias caused by the telescope. Of course, additional measurements in various conditions would be of great interest to confirm this conclusion. In particular, simultaneous measurements from WFS and DIMM on the same source could be performed to consolidate the hypothesis of a bias caused by the telescope itself.

The phase variance estimated along the pass gives an idea of the fluctuations of the zenithal Fried parameter, as estimated with Eq. (1), during $210 \mathrm{~s}$, for an elevation angle from $15^{\circ}$ to $43^{\circ}$. It appears that the variability of the zenithal Fried parameter, estimated by the standard deviation of the normalized difference between the biased $r_{0}$ from GDIMM and the measured $r_{0}$, does not exceed $10 \%$ on the whole trajectory.

\section{$3.2 \mathrm{Cn}^{2}$ profile estimation}

Temporal statistics of the measured wavefront are investigated here to assess the $\mathrm{C}_{\mathrm{n}}{ }^{2}$ profile distribution along the LOS. At a given elevation, the temporal Power Spectral Densities 
(temporal PSD) of the Zernike coefficients of same radial orders are added. The result is plotted in 0 for radial orders from 2 to 6 .

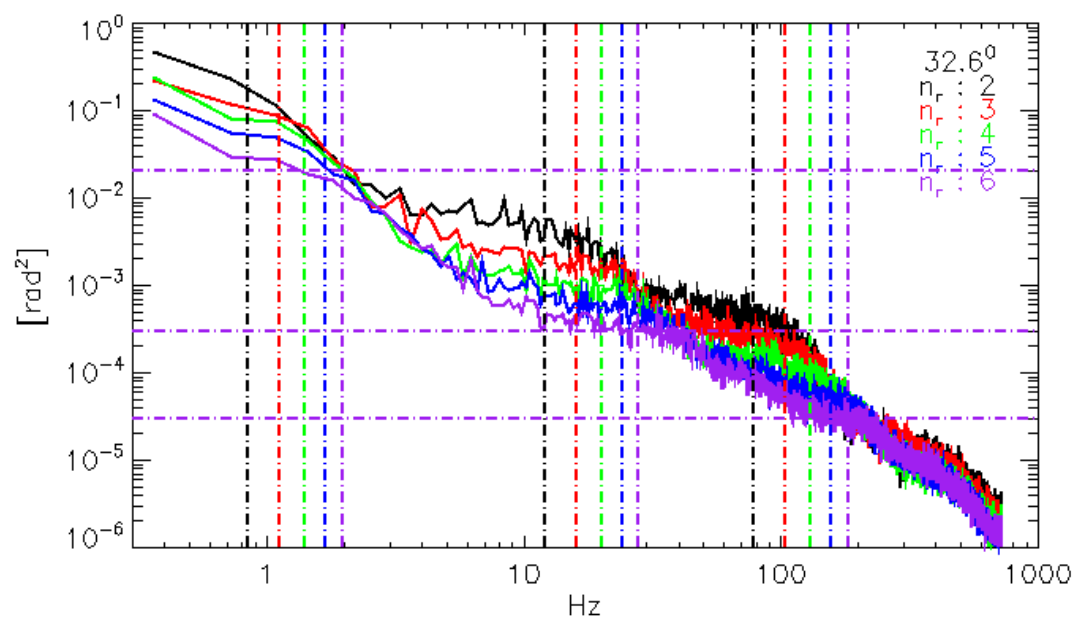

Fig. 8 Temporal spectra for radial orders from 2 to 6 for the $32.6^{\circ}$ elevation. Cutoff frequencies for each radial orders are reported (vertical mixed color lines). 
The shape of the temporal PSD per radial order gives an insight into the temporal correlations of the wavefront. These correlations can be related to the turbulence structure along the LOS owing to the apparent velocity of the satellite and assuming Taylor frozen flow hypothesis ${ }^{18}$. For a given turbulent layer and a given polynomial, its spectrum asymptotically follows power laws. At high frequencies, the theoretical power law is the same for all radial order in $v^{-17 / 3}$ due to the telescope aperture filtering. This power frequency is hardly visible in practice due to noise and aliasing as the Zernike coefficients are reconstructed from wavefront sensor data. This results into less steep power law, with a frequency decrease that depends on the wavefront sensor configuration and on noise propagation, hence hardly determined. At low frequency the slope of the asymptote depends on the radial order. For $n_{r}=1$ the power law is in $v^{-2 / 3}$, for $n_{r}>1$ the power law is in $v^{0}$. The two regimes are separated by a cut-off frequency which is increasing with the radial degree of the polynomial according to ${ }^{18}$ :

$$
v_{\mathrm{c}, \mathrm{l}}=0,3\left(\mathrm{n}_{\mathrm{r}}+1\right) \frac{\mathrm{V}_{\perp}}{\mathrm{D}}
$$

where $\mathrm{D}$ is the telescope diameter and $\mathrm{V}_{\perp}$ the norm of the apparent velocity of the layer in the direction of the trajectory of the satellite. For multi-layer spectrum, the integration over a wide altitude range provides a broad wind velocity distribution. This means summing up spectra with fairly different cut-off frequencies. This results into smooth transitions between the two asymptotic regimes. From these considerations it is possible to identify into the Zernike temporal PSD for $n_{r}>2$ three zones showing a smooth transition between two asymptotic regimes $\left(v^{0}\right.$ for low frequencies, steeper for higher frequencies) corresponding to three main layers. These three zones are illustrated in Fig. 9 for the case $n_{r}=6$. The asymptotes intersect at $v_{c, 1}=1,5 \mathrm{~Hz}, v_{c, 2}=$ 
$28 \mathrm{~Hz}$ and $v_{\mathrm{c}, 3}=190 \mathrm{~Hz}$ respectively, reported in the figure with vertical purple mixed lines.

From these frequencies three major layers are identified.

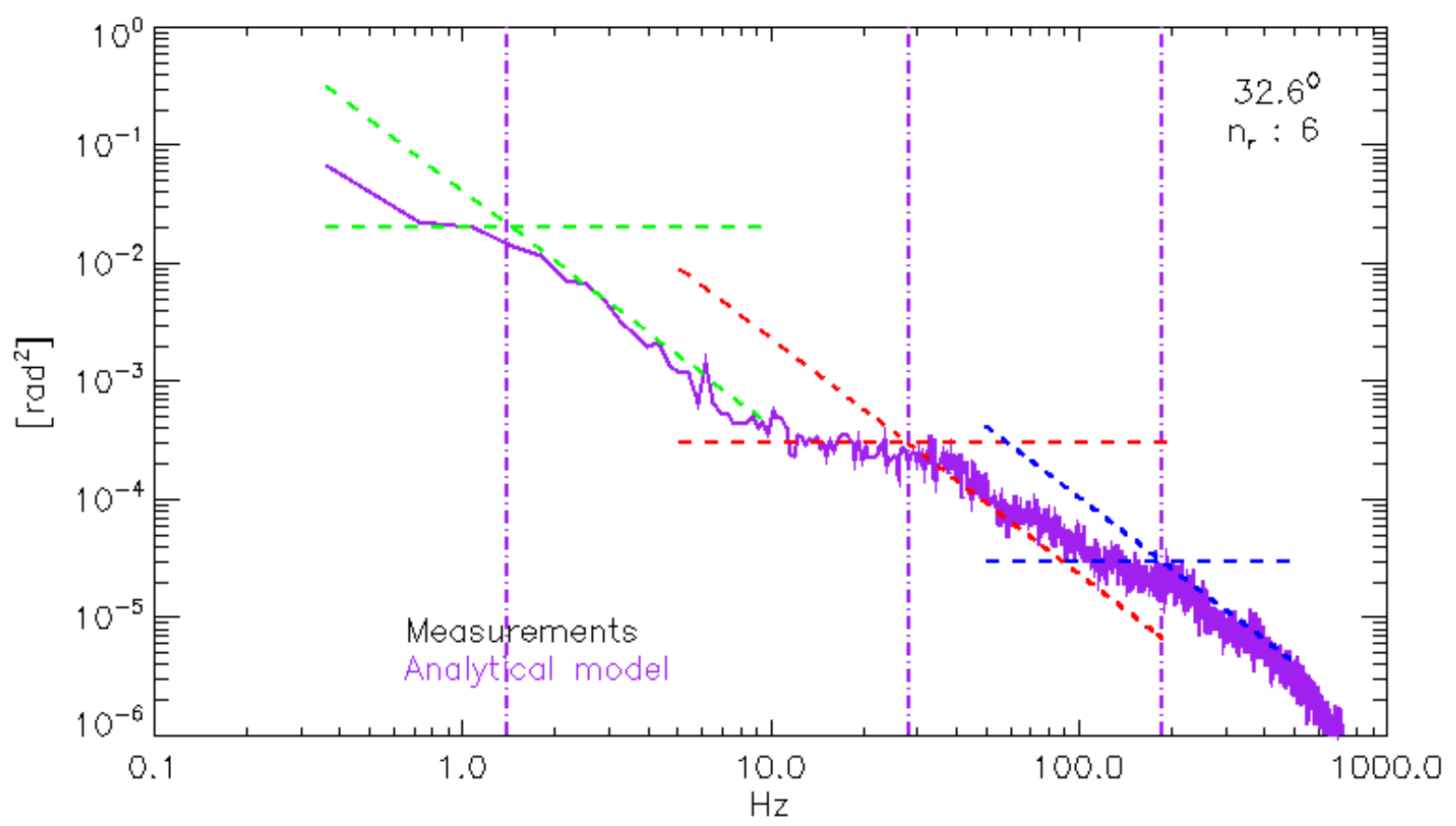

Fig. 9 Temporal spectra for radial order 6 for the $32.6^{\circ}$ elevation. Asymptotes enabling the identification of the three main layers are reported. Green: loaw altitude layer, red: intermediate layer, blue: high altitude layer. Vertical mixed color lines: frequencies corresponding to each layer.

The corresponding apparent velocities, obtained by inverting (3), are respectively, $\mathrm{V}_{\perp, 1}=1 \mathrm{~m} / \mathrm{s}$, $\mathrm{V}_{\perp, 2}=20 \mathrm{~m} / \mathrm{s}, \mathrm{V}_{\perp, 3}=133 \mathrm{~m} / \mathrm{s}$. The first cut-off frequency is slightly visible for radial order 6 . Its existence is consolidated by the analysis of the smaller radial orders where the cut-off frequencies computed from Eq. (3) and $\mathrm{V}_{\perp, 1}$ are reported in Fig. 8 (mixed vertical color lines).

The major contributor to apparent velocity of the turbulent layers may be attributed to the satellite displacement. Natural wind is therefore neglected in the following. It is possible to link the apparent layer velocity with the layer distance from the telescope and therefore with the layer 
height: from basic trigonometric considerations, the altitude of the turbulent layer responsible for PSD cut-off frequency is approximated by:

$$
\mathrm{h}_{1}=\frac{\mathrm{V}_{\perp, 1}}{\omega} \sin \theta
$$

where $\omega$ is the angular velocity of the telescope. Hence, the obtained altitudes of the three turbulent layers are $\mathrm{h}_{1}=100 \mathrm{~m}, \mathrm{~h}_{2}=1.3 \mathrm{~km}$ and $\mathrm{h}_{3}=12 \mathrm{~km}$.

A Multi-Gaussian (MG) profile is built from this three layer decomposition in order to check if considering only a few layers is sufficient to account for Zernike modes temporal PSD. The profile is composed of three Gaussian layers located at altitude $h_{k}$ of thickness $\sigma_{k}$ and turbulence strength $\mathrm{C}_{\mathrm{n}}^{2}\left(\mathrm{~h}_{\mathrm{k}}\right)$ :

$$
\mathrm{C}_{\mathrm{n}}^{2}(\mathrm{~h})=\sum_{\mathrm{k}=1}^{3} \mathrm{C}_{\mathrm{n}}^{2}\left(\mathrm{~h}_{\mathrm{k}}\right) \exp \left[\left(\frac{\mathrm{h}-\mathrm{h}_{\mathrm{k}}}{\sigma_{\mathrm{k}}}\right)^{2}\right]
$$


The height $\left(\mathrm{h}_{\mathrm{k}}\right)_{\mathrm{k}=[1,2,3]}=[0,1.3,12.4] \mathrm{km}\left(\sigma_{\mathrm{k}}\right)_{\mathrm{k}=[1,2,3]}=[0.1,3,3] \mathrm{km}$ and $\left(\mathrm{C}_{\mathrm{n}}{ }^{2}\right)_{\mathrm{k}=[1,2,3]}=\left[6,110^{-15}\right.$, $\left.710^{-17}, 310^{-17}\right] \mathrm{m}^{-2 / 3}$ are provided by the temporal PSD analysis.

The $\mathrm{C}_{\mathrm{n}}^{2}$ profile estimated from WFS data analysis is plotted in Fig. 10 and compared with a typical Hufnagel-Valley model. The $\mathrm{C}_{\mathrm{n}}{ }^{2}$ at ground level of $\mathrm{H}-\mathrm{V}$ model is adapted so that the two profiles present the same Fried parameter (here $10 \mathrm{~cm}$ along the $\operatorname{LOS}$ at $\lambda=976 \mathrm{~nm}$ ).

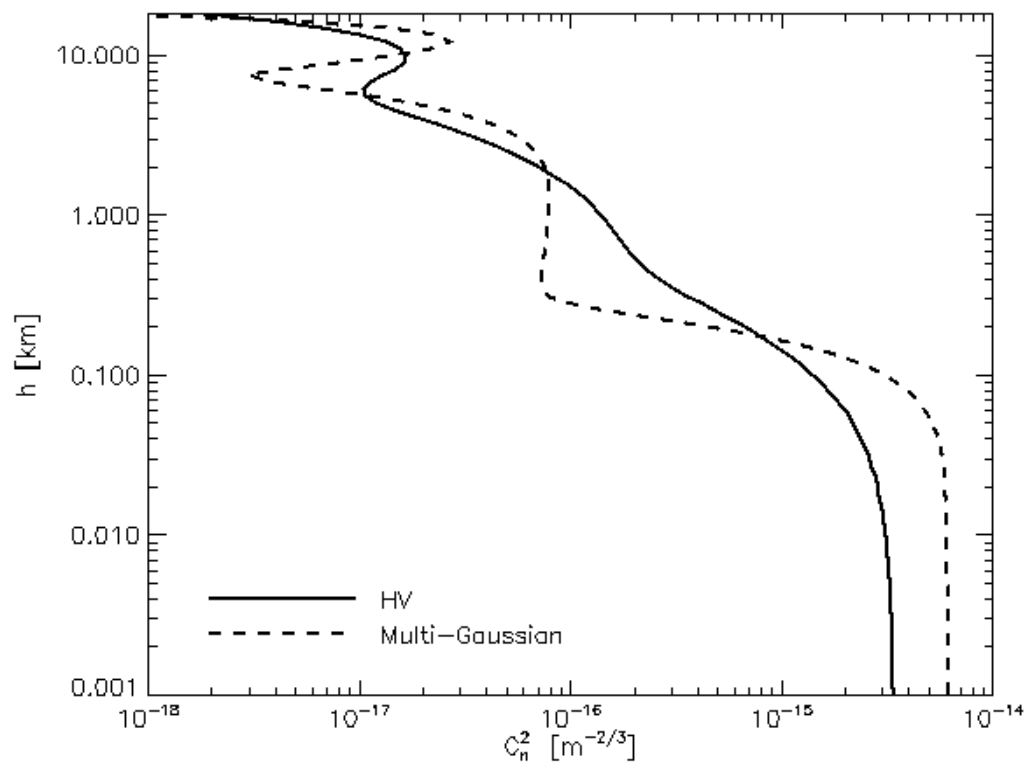

Fig. 10 Custom $C_{n}^{2}$ (Multi-Gaussian) and Hufnagel - Valley profiles. 
The turbulence strength of high altitude layers and their thickness have been chosen to fit scintillation index temporal spectrum with the results of the analytical model described in ${ }^{19}$ for the highest elevations. Scintillation index is computed from the WFS intensities recorded in each subaperture. For each data-set, the normalized intensity fluctuations $i_{k, j}$ per subaperture are determined according to:

$$
\mathrm{i}_{\mathrm{k}, \mathrm{j}}=\frac{\mathrm{I}_{\mathrm{k}},{ }_{\mathrm{j}}}{\frac{1}{\mathrm{~N}_{\mathrm{j}}} \sum_{\mathrm{k}=1}^{\mathrm{N}_{\mathrm{j}}} \mathrm{I}_{\mathrm{k}, \mathrm{j}}},
$$

where the subscript $\mathrm{j}$ stands for the data set and $\mathrm{k}$ corresponds to the kth measurement in the data-set. $\mathrm{Nj}$ is the number of samples in each data set $(\mathrm{Nj}=20$ kilo-samples here $)$. The temporal PSD of the scintillation index and results of the analytical model are both plotted in Fig. 11 for the previously studied elevations. For elevations $28.2^{\circ}$ and above, the analytical model fits well the data. For elevation below $25^{\circ}$ no satisfying agreement was found due to significant influence of pupil stabilization that was turned off before the AO loop was closed.

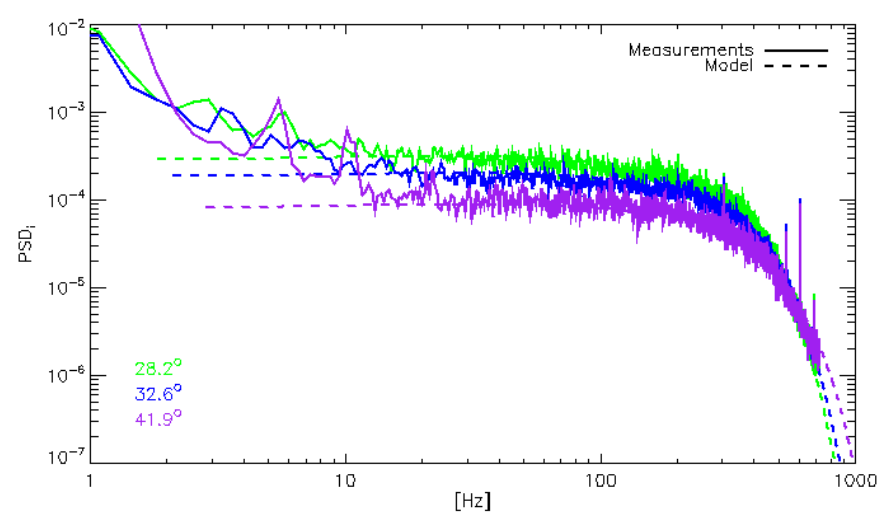

Fig. 11 Flux fluctuations power spectral densities for $28.2^{\circ}, 32.6^{\circ}$ and 41.9 . 


\subsection{Consistency of the Cn2 profile with measurements}

\subsubsection{Wavefront characteristics}

To evaluate the pertinence of the analysis, the MG model has been used as input of an analytical model of radial temporal PSD taken from ${ }^{18}$. The Zernike phase variance spectrum obtained from the MG model for radial orders 2 to 6 is plotted in Fig. 12, as well as the spectrum obtained from the data. The low frequencies are badly fitted due to the presence of slowly varying aberrations that are not taken into account in the model. For higher frequencies the model adequately fits the data.

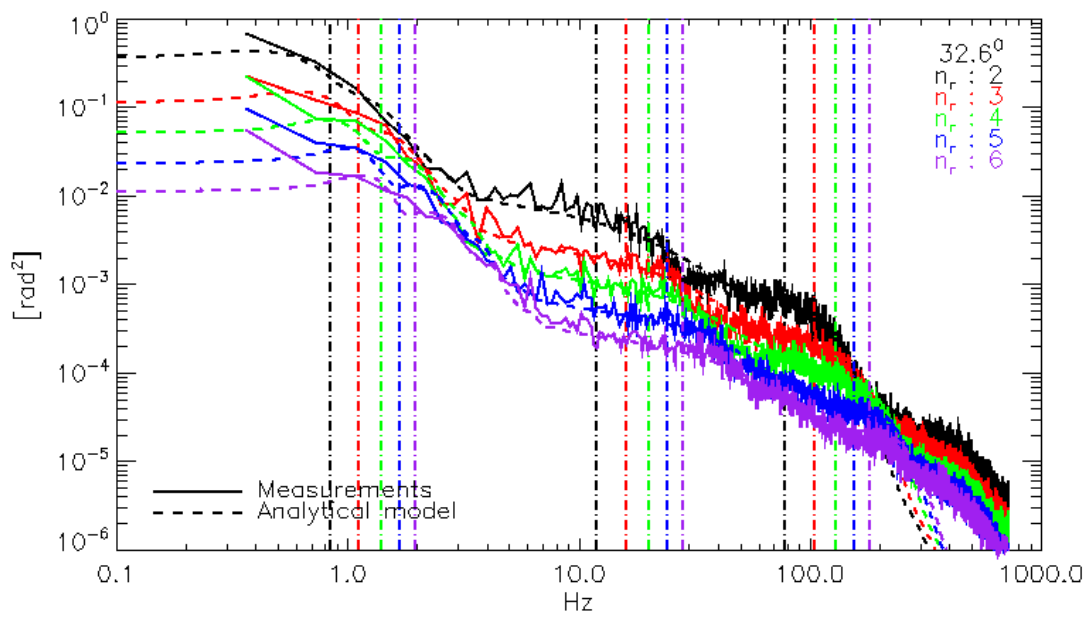

Fig. 12- Results of analytical model compared to temporal PSD for radial orders 2 to 6 for $32.6^{\circ}$ elevation.

The richness of wavefront data is illustrated here as they enable the estimation of turbulence parameters with a rather straightforward approach. The same approach could be applied to the whole set of data to obtain an insight into the variability of turbulence profiles along the trajectory of the satellite. A preliminary trend can be spotted by considering the evolution of temporal power spectrum as a function of elevation. The measured temporal PSDs of Zernike coefficients of radial order 6 are plotted in Fig. 13 for increasing elevations. Between $28.2^{\circ}$ and $41.9^{\circ}$, the temporal PSDs present the same shape; the cutoff frequencies attributed to high 
altitude layers are similar. It appears here that the signature of high altitude layers very slightly depends on elevation on a range close to $11^{\circ}$ : in this angular interval, turbulence distribution along the LOS seems almost constant regarding the considered criterion. This gives an order of magnitude for the required spatial precision of the knowledge of the turbulence profile necessary for performance estimation. From this elevation range and basic trigonometric considerations the longitudinal extension of the stationarity zone, ie the zone where $\mathrm{C}_{\mathrm{n}}{ }^{2}$ is considered as constant, of the turbulent layer can be computed. The principle of the computation is illustrated in Fig. 14. Between $28.2^{\circ}$ and $41.9^{\circ}$ we find $2.2 \mathrm{~km}$. This means that a physical model able to predict the optical turbulence statistics with a typical resolution of few kilometers could be considered as an interesting starting point for performance prediction, at least in this elevation range, and considering the shape of the temporal PSDs is the relevant metric to assess AO correction efficiency.

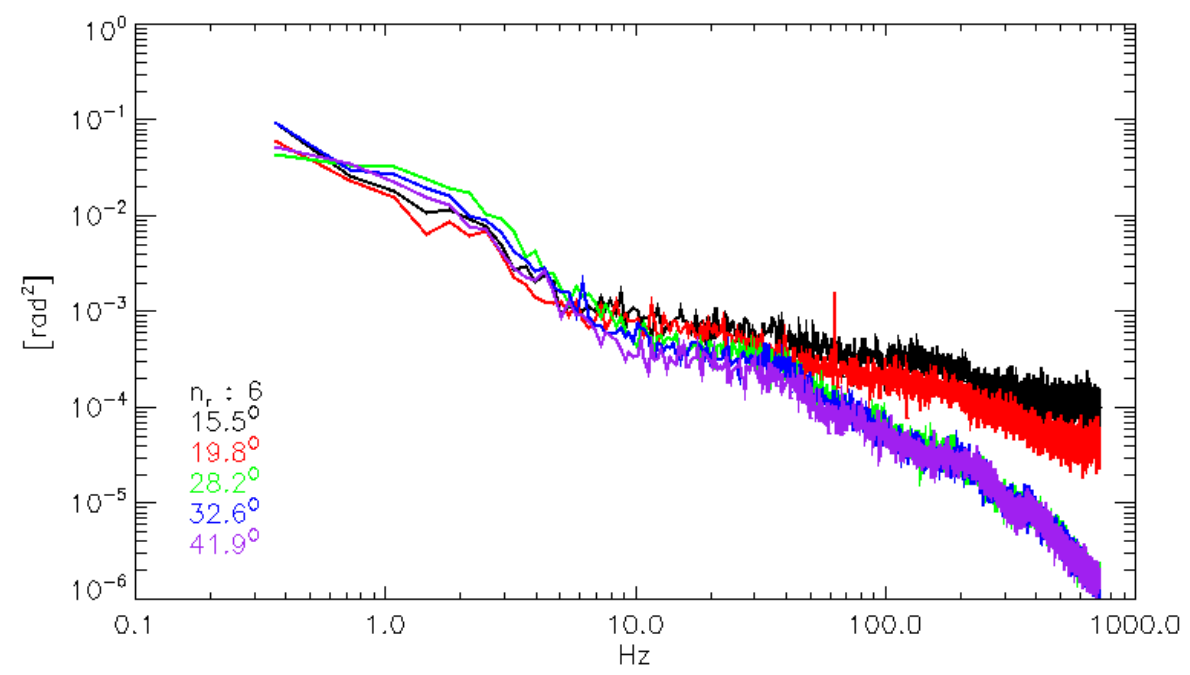

Fig. 13 Evolution of temporal PSD of sixth radial order for increasing elevation. 


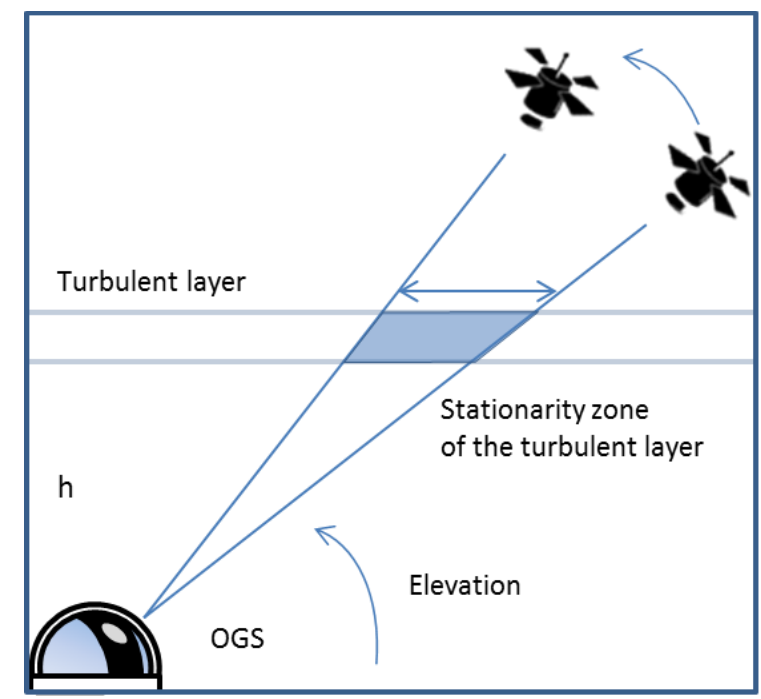

Fig. 14 Longitudinal extension of the stationarity zone of the turbulence layer located at altitude $h$.

\subsubsection{Scintillation characteristics}

We are comparing here the results of scintillation index evaluation from SH-WFS data to the results expected from the MG profile. Scintillation index estimation from SH-WFS data must be performed with caution as during the acquisition of the data set ( 13 seconds) some of the acquisition parameters may vary. For instance, before turning on the pupil stabilization (when AO loop is closed), the position of the telescope aperture projected in the WFS lenslet array may be shifted up to a half of the size of a microlens. For this reason, only unocculted subapertures are exploited to compute the scintillation index. Moreover to overcome the power fluctuations delivered by the laser source or other sources of low frequencies fluctuations, the scintillation index is evaluated from the integral of the temporal power spectral density after filtering fluctuations below $30 \mathrm{~Hz}$. The evolution of the scintillation index measurement as a function of elevation is plotted in Fig. 15. The results of scintillation index estimation from the Multi Gaussian $\mathrm{C}_{\mathrm{n}}{ }^{2}$ profile is also reported in the same figure (dashed red line). Below $25^{\circ}$, the acquisition parameters were modified to optimize the AO loop performance and pupil 
stabilization was off, so scintillation index evaluation is not correct. For elevations above $26^{\circ}$, the parameters were stabilized. The measured scintillation index decreases with the elevation as it is expected by theory. The MG profile correctly fits the measurements. The discrepancy between the scintillation index obtained from the data and the one obtained from the analytical model does not exceed $7 \%$.

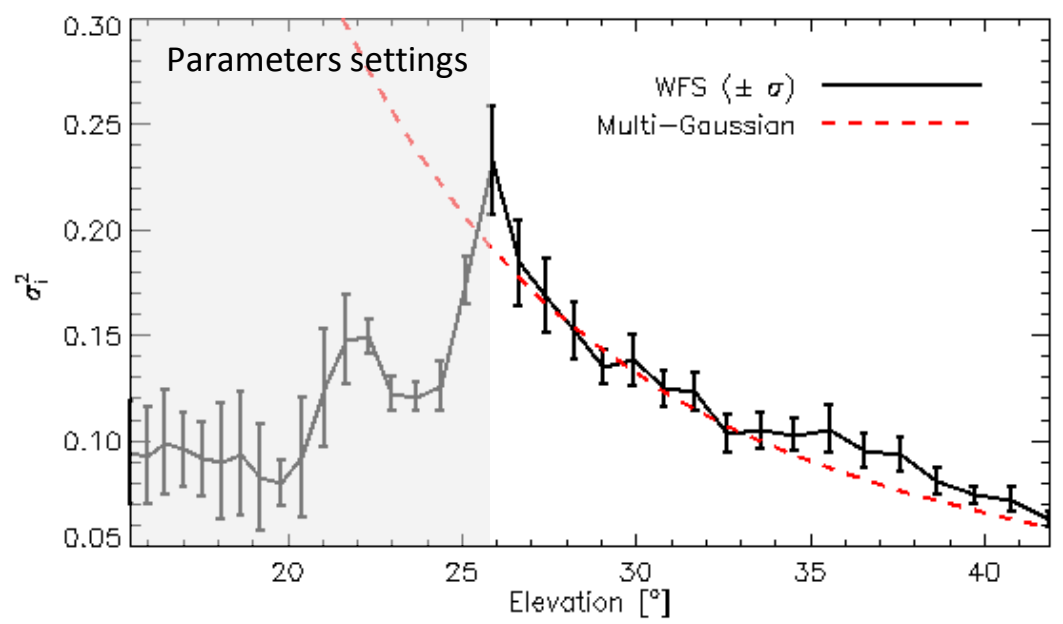

Fig. 15 Evolution of the scintillation index as a function of elevation from measurements (black), and from multigaussian profile (dashed red).

\section{Analysis of AO Performance}

In the following AO results are presented and discussed. We first present global AO performance then discuss more specifically $\mathrm{AO}$ performance in the context of satellite to ground telecommunication. The quantity of interest for high data rate laser link is the single mode fiber Coupling Efficiency $(\mathrm{CE})^{20,21}$. Experimentally, CE could be evaluated provided that injection into a single mode fiber is performed, or, that the residual wavefront is perfectly known, allowing a numerical computation. In the present case, no single mode fiber could be placed at the focus of the AO system. As mentioned before, the system was not designed for optical data transmission and it is not currently adapted to injection into single mode fiber, with a f-number 
of 32. Consequently, CE could not be evaluated. We propose to evaluate a pseudo-coupling efficiency, related to Power In the Bucket (PIB). We validate this criterion compared to CE by simulation then discuss experimental results of PIB.

\subsection{Closed loop results}

Closed-loop data are considered after $\mathrm{AO}$ and pupil loops are stabilized. Pupil stabilization clearly provides a gain in performance due to telescope pupil drift with respect to Coudé Focus during fast steering of the telescope, inducing possible loss of edge subapertures.

Fig. 16 shows long exposure images obtained either with or without AO correction. These images are computed as time average of 256 frame sequences. Similar scaling is used on the images leading to hardly visible image without $\mathrm{AO}$, despite an exposure time 10 times greater without AO (100 ms) than with AO (10 ms). Accounting for this exposure time difference the gain in maximum intensity is $45(16 \mathrm{~dB})$. Gain in Full Width at Half Maximum (FWHM) between open and closed loop image is of nearly 7.

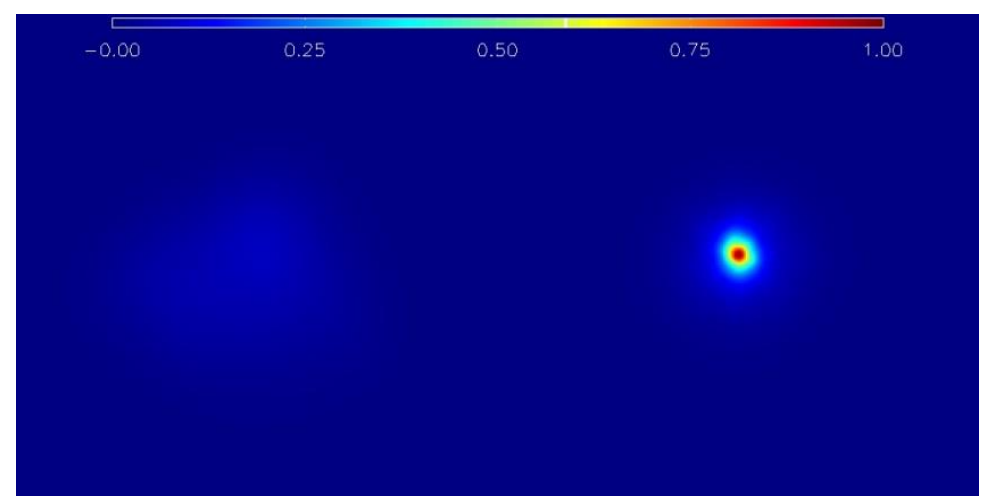

Fig. 16 Long exposure images (linear scale) obtained in open (left) or closed AO loop (right), with same scaling. Open-loop image is hardly visible, due to a factor 45 in maximum intensity and $\sim 7$ in FWHM. 


\subsection{Evaluation of a pseudo coupling efficiency}

\subsubsection{Derivation of a pseudo coupling efficiency criterion}

Considering a single mode fiber at the focal plane of an optical system, coupling efficiency is computed either in the pupil or focal plane ${ }^{20,21}$, and in the latter case takes the form ${ }^{21}$ :

$$
\rho=\left[\frac{1}{2} \int \widetilde{\boldsymbol{E}}_{x}\left(\frac{r}{\lambda f}\right) \times \boldsymbol{H}_{y}^{*}\left(\frac{\boldsymbol{r}}{a}\right) \hat{z} d \boldsymbol{r}\right]^{2},
$$

where $a$ is the fiber core radius, $\lambda$ the wavelength, $f$ the focal length, $\boldsymbol{E}(\boldsymbol{r})$ the electric field transmitted at the pupil and $\widetilde{\boldsymbol{E}}(\boldsymbol{r})$ its Fourier transform with:

$$
\boldsymbol{E}(\boldsymbol{r})=E_{0} \boldsymbol{\Psi}(\boldsymbol{r}) \boldsymbol{P}(\boldsymbol{r})
$$

$E_{0}$ is the field amplitude, $\Psi(\boldsymbol{r})=\exp (i \varphi(\boldsymbol{r})) \hat{z}$ the phase and direction of the wavefront and $\boldsymbol{P}(\boldsymbol{r})$ the transmittance of the pupil. $\boldsymbol{H}_{y}(\boldsymbol{r})$ is the magnetic field vector of the $\mathrm{LP}_{01}$ mode of the fiber. The integral is evaluated over the core and cladding region. Full description of $\boldsymbol{H}_{y}(\boldsymbol{r})$ and details of the calculation is provided $\mathrm{in}^{21}$.

Assuming a perfect match between optical system output and single mode fiber numerical apertures, no turbulence, and no central obscuration, the maximum CE can be evaluated to $\rho_{0}=80 \%^{20}$, corresponding to the overlap between hard spherical aperture with plane wave front and Gaussian mode. As mentioned before, CE could not be estimated on the bench in absence of fiber injection. As an alternative, we consider short exposure images obtained in closed loop and derive a pseudo coupling efficiency. Fig. 17 presents typical short exposure images obtained either in open or closed loop, this time each image being normalized with respect to its maximum for better illustration. Impact of AO correction is clearly visible.

The pseudo coupling efficiency is evaluated from short exposure images assuming an equivalent single mode fiber which numerical aperture matches with the diffraction limited PSF of the 
optical instrument (without turbulence). Pseudo coupling efficiency is computed from intensity distribution in the focal plane: it comes down to calculation of the overlap between the system PSF and the fiber Gaussian mode intensity profile. Thus, assuming Gaussian approximation, a 1/e $\mathrm{LP}_{01}$ mode radius $\omega$ is derived ${ }^{20}$ and illustrated in Fig. 17 as a centered fixed white circle (corresponding to $1 / \mathrm{e}$ radius on intensity profile).

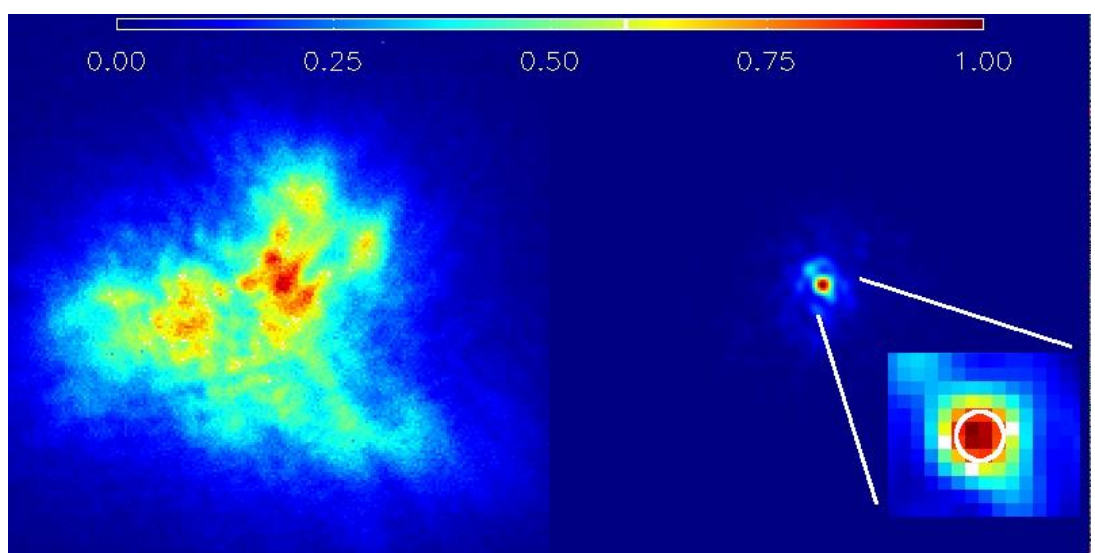

Fig. 17 Short exposure images (linear scale) obtained in open (left) or closed AO loop (right). For better visualisation, both images have been normalized to their maximum. On right image a zoom has been proposed to represent (white circle) the equivalent fiber Gaussian mode intensity with $\omega$.

Noting $\boldsymbol{I}_{\mathbf{1}}=\left|\widetilde{\boldsymbol{E}}\left(\frac{\boldsymbol{r}}{\lambda f}\right)\right|^{2}$ and $\boldsymbol{I}_{\mathbf{2}}$ the Gaussian mode intensity distribution in focal plane, and assuming that $\boldsymbol{I}_{\mathbf{1}}$ and $\boldsymbol{I}_{\mathbf{2}}$ are normalized so that $\int I_{1}(\boldsymbol{r}) d \boldsymbol{r}=\int I_{2}(\boldsymbol{r}) d \boldsymbol{r}=1$, the pseudo coupling efficiency $\bar{\rho}$ is computed as:

$$
\bar{\rho}=\int I_{1}(\boldsymbol{r}) I_{2}(\boldsymbol{r}) d r
$$

where integration is performed over the field. For a diffraction limited spot (airy pattern) $\bar{\rho}=$ $\bar{\rho}_{0}$.

Obviously, our pseudo coupling efficiency, while adapted to the present experimental set-up, does not correspond to true CE (defined in Eq. (7)). It is more related to a Power In the Bucket (PIB) or encircled energy criterion, assuming a weighting map (the Gaussian intensity 
distribution). Pragmatic and simple, it is also independent from optical throughput and only depends on intensity spatial distribution. Still, as we will show in simulation, it also provides a fair estimate of true coupling efficiency.

In the following, coupling efficiency and PIB, as defined previously, will be compared after normalization with respect to their diffraction limited value (resp. $\rho_{0}$ and $\bar{\rho}_{0}$ ) for coherence.

\subsubsection{Experimental evaluation of PIB}

PIB is evaluated from open and closed loop short exposure images. In open loop, the PIB proves to be meaningless, considering the shape of the Point Spread Function (PSF) and the $100 \mathrm{~ms}$ exposure time that leads to time smoothing. Still a $0.6 \%$ value can be estimated. In closed loop, an $8.8 \%$ mean value is obtained, with a $3 \%$ rms. Time evolution of PIB is proposed in Fig. 18. Losses of coupling (in PIB) down to $3 \%$ are still visible.

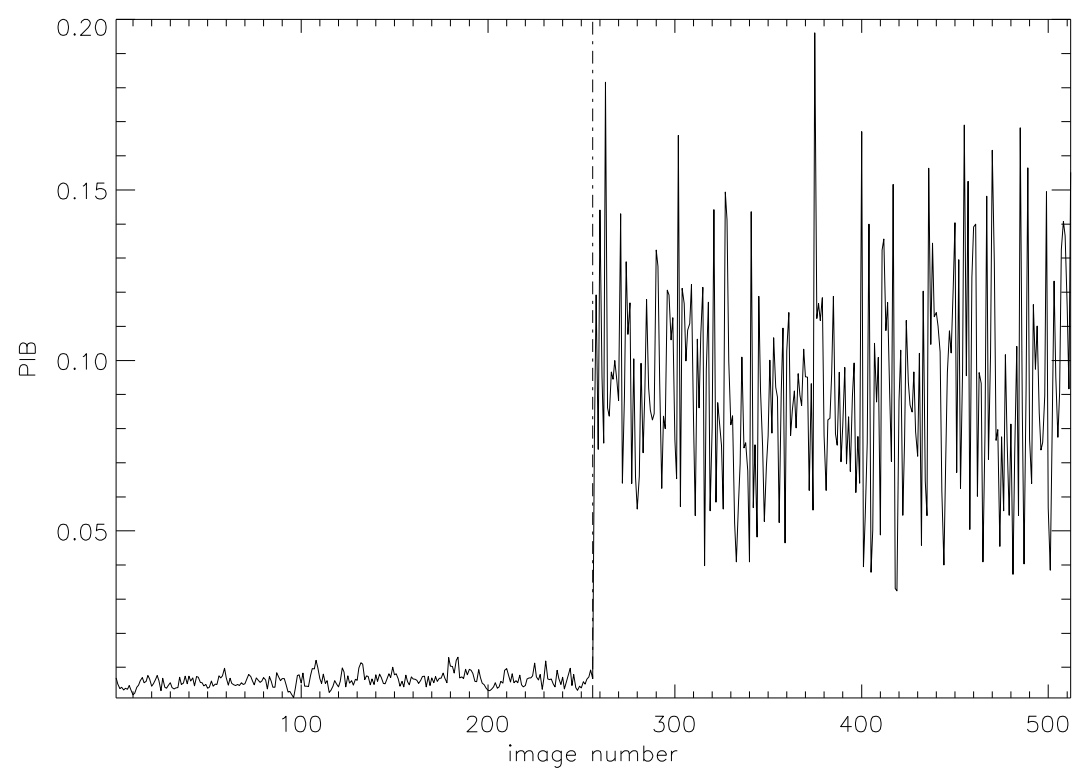

Fig. 18 Time evolution of PIB along frame number, first in open then in closed loop (dashed line delimits the separation). 


\subsection{Comparison to numerical simulation results}

The experimental results are now compared to the expectable performance of the AO bench obtained with a numerical simulation. This simulation is based on a pseudo-analytical model described elsewhere ${ }^{22}$ able to deliver correlated time series of $\mathrm{AO}$ corrected wavefront. It is parametrized to take into account the $\mathrm{C}_{\mathrm{n}}{ }^{2}$ profile estimated from the experiment and the real $\mathrm{AO}$ bench characteristics (DM/WFS characteristics, loop delay...). The simulation tool provides residual wavefronts but also focal plane images. Thus, PIB can be estimated in the same way as the experiment (focal images based). Rigorous computation of CE based on the the knowledge of the complex field. We first address numerical comparison of $\mathrm{CE}$ and PIB to validate our approximation then discuss comparison of experimental results with simulated ones.

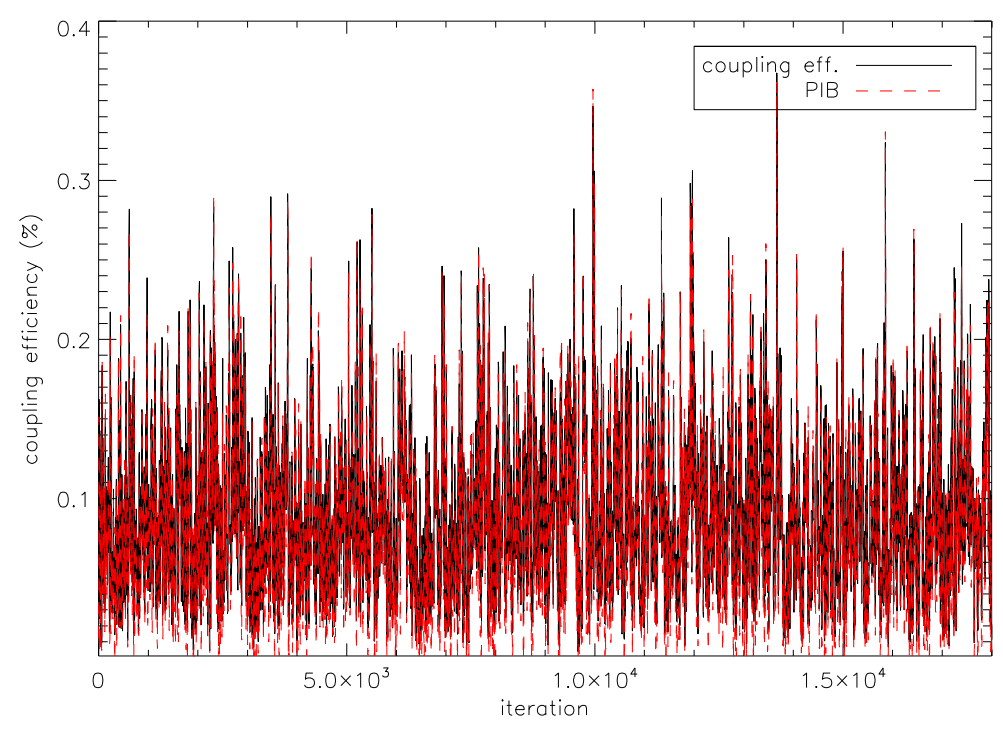

Fig. 19 Time evolution of PIB along frame number, compared to coupling efficiency, both obtained in numerical simulation.

\subsubsection{Numerical validation of PIB}

We focus here on the numerical validation of PIB compared to CE. The numerical focal plane intensity distribution is used to derive PIB following Eq. (9) while the corresponding residual wavefront is used to derive CE using Eq. (7). Result over time is proposed in Fig. 19. A very 
good matching is found, PIB slightly over-estimates CE (less than 0.7 point in average) and difference between the two is less than 1 point root mean square, confirming that PIB is an acceptable estimate of CE.

\subsubsection{Analysis of experimental PIB}

We now use numerical simulation, obtained in representative conditions of the experimental ones, to confront experimental measurements. First, average value of PIB proves to be coherent between experiment and numerical simulation with respective values of $8.8 \%$ and $8.2 \%$. This demonstrates the accuracy of the numerical simulation and consistency of experimental results. Furthermore numerical simulation provides access to full frame rate $(1.45 \mathrm{kHz})$ images and thus the experimental PIB temporal PSD (limited to the imaging camera frame rate of $100 \mathrm{~Hz}$ ) can be compared to the simulated one (1.45 kHz) (see Fig. 20).

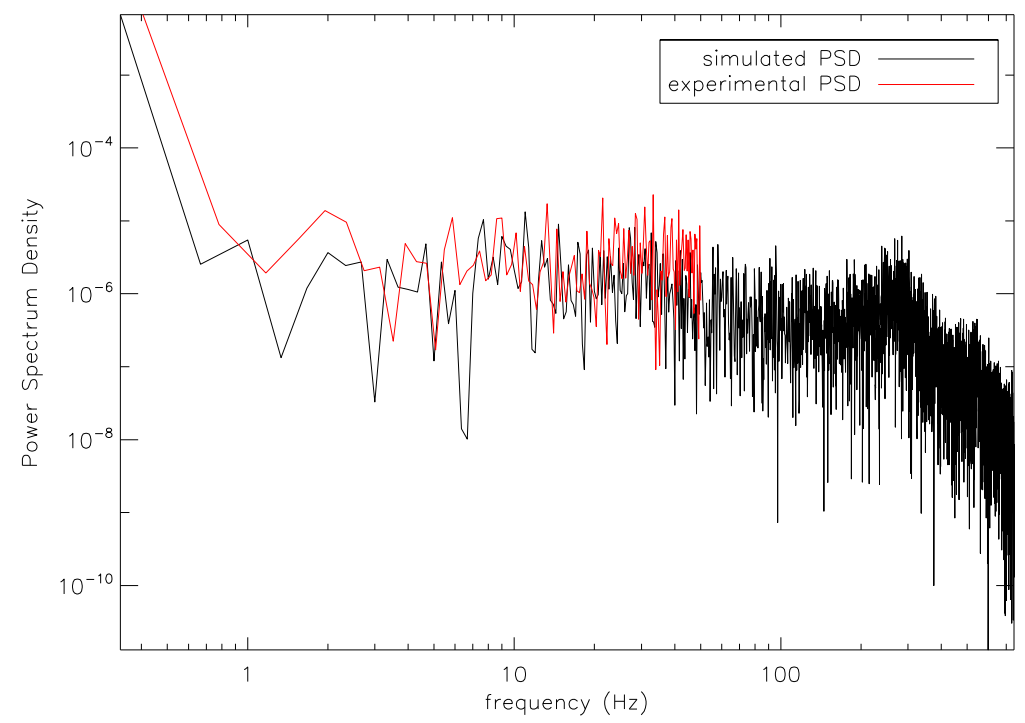

Fig. 20 Compared temporal PSD of PIB as obtained experimentally (at $100 \mathrm{~Hz}$ sampling frequency) in red or by numerical simulation. A good matching is obtained in the accessible frequency range.

A very good matching is obtained up to $40 \mathrm{~Hz}$, indicating a correct temporal behavior. The 40 $\mathrm{Hz}$ to $50 \mathrm{~Hz}$ range may be affected by temporal aliasing. Acquisition with smaller exposure time 
or a faster detector is now under consideration to extend this cross-check in the future version of the AO bench.

Finally, image acquisition process on PCO camera with a $10 \mathrm{~ms}$ exposure time (closed-loop) can also be accounted for in simulation, so as to take into account possible bias in PIB estimation due to turbulence residuals averaging and PSF blurring. In Fig. 21 left probability densities of PIB for experimental data and simulated ones are compared in this case. Probability density of CE in numerical simulation with $10 \mathrm{~ms}$ time averaging is also proposed. Very similar distributions are found, with the $8.2 \%$ average performance in simulation against $8.8 \%$ in experiment mentioned earlier. CE still exhibits a slightly lower value as mentioned before, with an average value of $8.15 \%$.
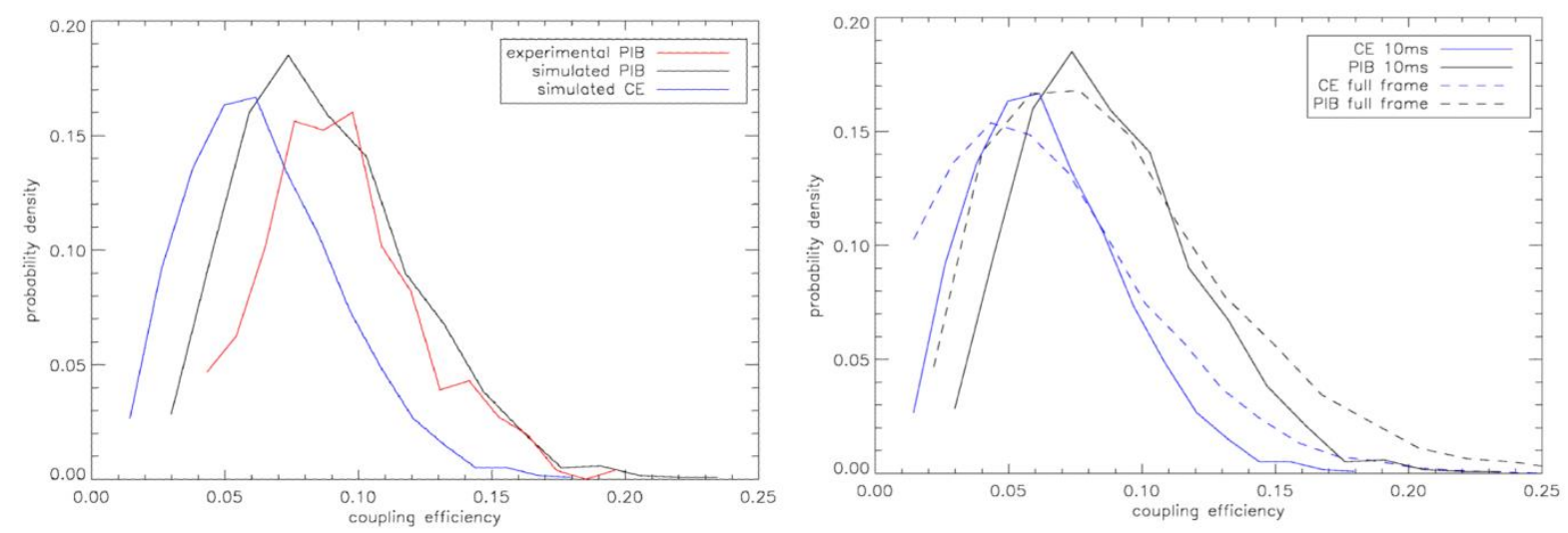

Fig. 21 Left: compared statistics (probability density) of PIB as obtained experimentally (red) or by numerical simulation (black). CE obtained in simulation with similar time averaging $(10 \mathrm{~ms})$ is added in blue. Right: statistics of PIB (black) and CE (blue) as obtained in numerical simulation for full frame rate $(1.45 \mathrm{kHz}$ ) (dashed line) or simulated $10 \mathrm{~ms}$ time averaging (exposure time) (solid line).

Fig. 21 right focuses on impact of time averaging. Only numerical simulation outputs are considered. PIB and CE probability densities, at full frame rate or with $10 \mathrm{~ms}$ time averaging (corresponding to exposure time), are proposed. In any case, PIB slightly overestimates CE and time averaging has marginal effect: mean value of $\mathrm{CE}$ is barely affected while dispersion (or variance) is slightly reduced due to natural smoothing induced by temporal averaging. 


\subsubsection{Experimental versus simulated AO performance}

Finally, the relevancy of the simulation is compared to the closed-loop residual error evaluation on the whole trajectory as a function of the time since the loop closure (Fig. 22). Corresponding elevation is reported on the top abscissa. These errors are computed by quadratic summation of the residual slopes measured by the WFS in closed-loop and projected onto Zernike modes. Performance is thus estimated over time period of $10 \mathrm{~s}$. Time interval for image acquisition and PIB estimation is indicated with vertical mixed lines. Simulated results are obtained using the estimated $\mathrm{C}_{\mathrm{n}}^{2}$ profile and the estimated contribution of the telescope (see 3.1 for bias evaluation). During the first $20 \mathrm{~s}$, performance increases slowly while AO loop is optimized (loop gain, EMCCD gain) and pupil stabilization converges. Impact of scintillation still needs to be assessed. Then performance is roughly stable, with a slow deterioration of performance becoming steeper after $38^{\circ}$. Simulated results fit well experimental ones between $27^{\circ}$ and $38^{\circ}$. Below $27^{\circ} \mathrm{AO}$ optimization is not reproduced by the simulation. Above $38^{\circ}$, per mode analysis of the measurement points out the vibrations on tip-tilt modes as the major contributor to the increase. These vibrations are to be related to telescope steering, which accelerates with elevation. Between $27^{\circ}$ and $38^{\circ}$ the residual error is mainly dominated by temporal error (for $75 \%)$.

These results, though encouraging, must be considered cautiously. While the global performance is clearly consistent and provides confidence in our ability to predict the $\mathrm{AO}$ approximate performance in realistic conditions, the simulation tool is still too simplistic to go further in the analysis. It indeed makes some simplifications such as assuming pure Kolmogorov turbulence, approximating DM controllable modes by pure Zernike modes, considering aliasing error as decoupled with temporal error (which may be discussed in case of fast turbulence layers), 
approximating the temporal error by a 3 frame delay control loop (instead of 3.3), assuming negligible influence of scintillation on wavefront sensing etc.

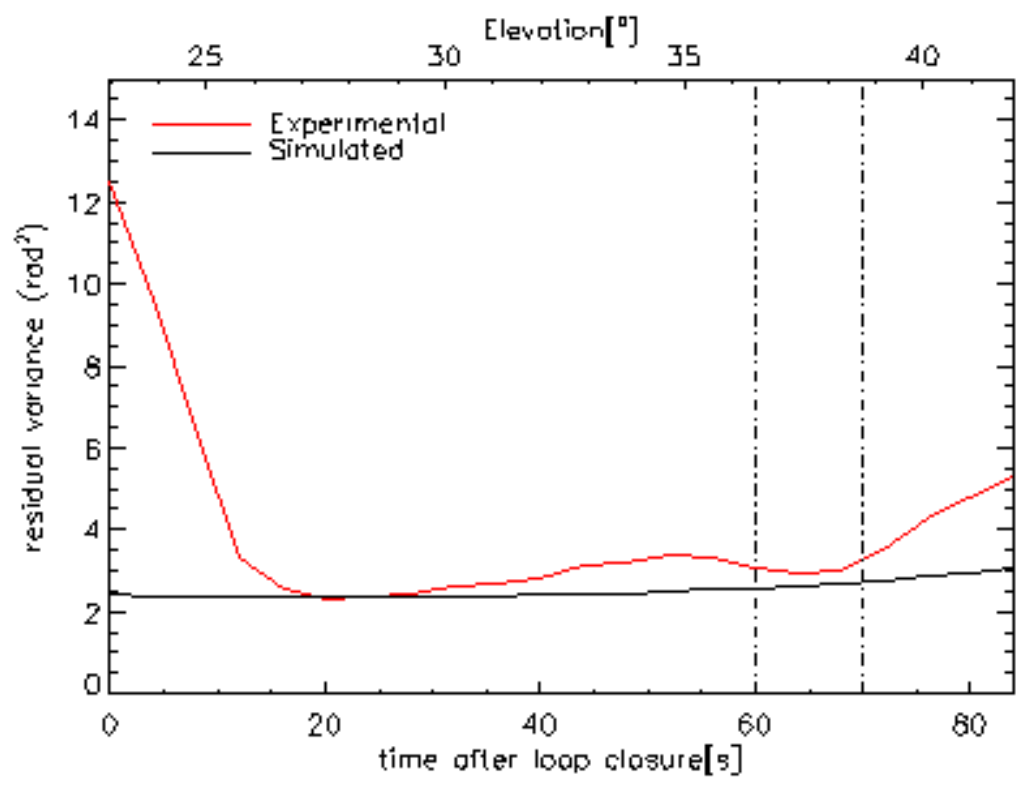

Fig. 22 AO loop performance in terms of wavefront variance $\left(\mathrm{rad}^{2}\right)$ as reconstructed from closed-loop slopes (Experimental, in red) and as estimated from the simulation (in black), at laser wavelength. Vertical dotted lines correspond to time interval for PIB estimation from PCO images.

These various limitations will contribute one way or the other to the final performance. In addition, though an extensive analysis of turbulence conditions has been performed based on the overall data acquired during the experiment, it is quite hard to ensure perfect consistency between simulation and experimental turbulence. Thus, some contributions still need to be assessed further. In particular, contribution of the Tip-Tilt modes requires particular attention, as residuals will strongly affect the final $\mathrm{CE}$. This can be understood considering first the time evolution of Tip (or Tilt) in open-loop as proposed in Fig. 23 and second the residual Tip (or Tilt) cumulated temporal PSD in closed loop in Fig. 24. First figure clearly shows a strong drift related to telescope residual pointing error. This contribution is clearly compensated in closedloop but Kolmogorov turbulence assumption shall probably be reconsidered. Second figure indicates that Tip (or Tilt) residual after AO loop closure is mainly affected by a $50 \mathrm{~Hz}$ vibration 
that falls unluckily at the $\mathrm{AO} 0 \mathrm{~dB}$ cut-off frequency, thus it is not corrected. In addition, this vibration will partly affect the PSF acquisition on PCO imaging camera as it works at $100 \mathrm{~Hz}$.

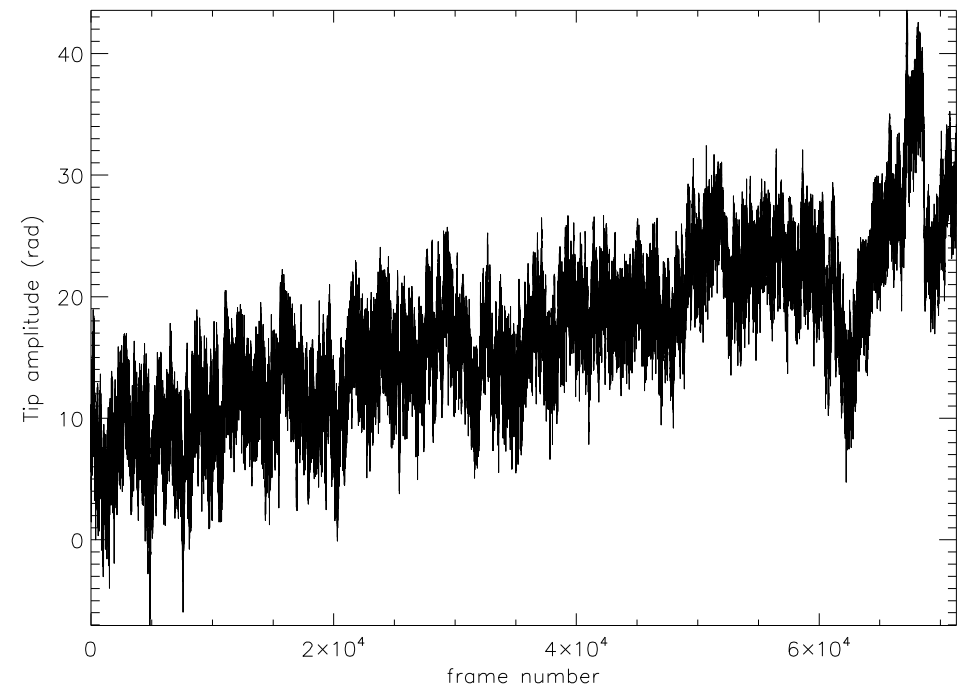

Fig. 23 Temporal evolution of tip mode in open loop. A clear drift is visible due to telescope residual pointing error.

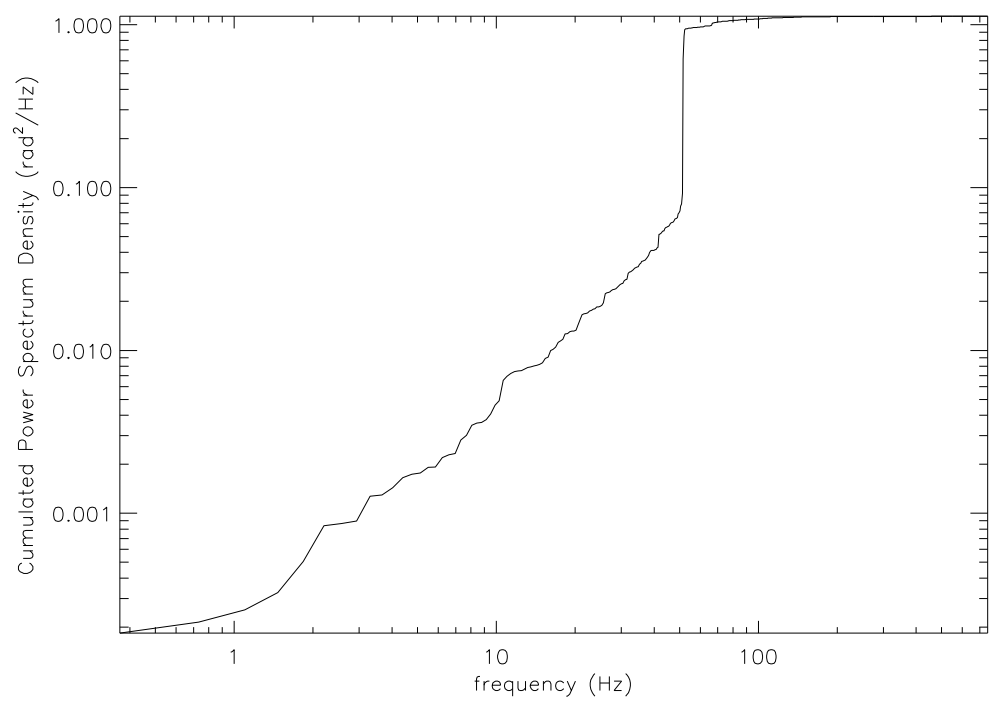

Fig. 24 Cumulated temporal Power Spectrum Density of tip mode in closed loop

As illustrated here, while global AO performance is well evaluated by the simulation tool, various contributors still need to be precisely assessed and accounted for in the simulation. Use of end-to-end simulation tools should be considered in the future, as they provide more detailed modeling of the system. Finally, the AO performance in terms of PIB has been estimated during 
a short period of time due to PCO imager time transfer. Instead, AO performance shall be evaluated on the overall closed-loop time and in the end using true injection into a single mode fiber.

\section{Conclusion}

We reported here demonstration of an AO corrected laser link at $976 \mathrm{~nm}$ established between the MéO telescope (Calern, France) and the SOTA terminal onboard SOCRATES micro-satellite. AO correction was continuously performed during more than $2 \mathrm{~min}$. This experiment provided extensive data and information on the turbulent conditions as well as a first evaluation of AO performance and limitations. Wavefront sensor data (slopes and intensities) were acquired during the satellite pass between $15.5^{\circ}$ and $41^{\circ}$ of elevation. First results of data reduction, as well as a comparison to analytical models, have been presented. We show that the measured $\mathrm{r}_{0}$ may be described using a unique $\mathrm{C}_{\mathrm{n}}{ }^{2}$ vertical profile including a bias to account for the local turbulence close to the telescope. The data analysis shows that, during this nighttime experiment performed on an astronomical site, without any wind measured at ground level, the Fried parameter was very stable during the pass. The standard deviation of the difference between the $r_{0}$ given by the measurement and the $\mathrm{r}_{0}$ given by the MG model was smaller than $10 \%$. The temporal analysis of the recorded wavefronts revealed the presence of three main turbulence layers on the LOS. A multi-Gaussian $\mathrm{C}_{\mathrm{n}}{ }^{2}$ profile has been proposed and parameterized with the measured data. It correctly accounts for wavefront temporal characteristics at the considered elevation. Analysis of , the MG model remains The Multi-Gaussian profile is also well adapted to fit the scintillation index evolution for elevations greater than $25^{\circ}$. As for Fried parameter, the scintillation index shows a striking constancy as its standard deviation is below $7 \%$ on a $15^{\circ}$ range. For a turbulent 
layer located at a $10 \mathrm{~km}$ altitude seen with a $35^{\circ}$ angle, this corresponds to a typical correlation scale greater than $8.2 \mathrm{~km}$. These numbers are consistent with the analysis of the temporal Zernike PSDs as a function of elevation that demonstrates that the $\mathrm{C}_{\mathrm{n}}{ }^{2}$ profile is almost constant between $28.3^{\circ}$ and $41.9^{\circ}$.

Further investigations are required to consolidate the conclusions for elevations below $25^{\circ}$. Wave optics simulations will be performed to investigate the effect of strong turbulence on the instruments. These elements are particularly significant in the design and automatization of AO systems for LEO to ground telecommunication, as they give clues on evolution of scintillation and Fried parameter along the path of the satellite and thus parametrization of AO.

In addition to this, the stationarity of turbulence conditions for several degrees of elevation tends to show that few kilometers lateral resolutions for the knowledge of vertical $\mathrm{C}_{\mathrm{n}}{ }^{2}$ profile might be sufficient for performance estimation along the trajectory. Of course these conclusions need to be consolidated by extensive measurements, with different turbulent conditions.

Regarding AO, continuous and stable loop closure has been demonstrated. Results of AO correction have been confronted to a pseudo-analytical simulation tool fed with the $C_{n}{ }^{2} M G$ profile estimated from the data. Estimated performance is consistent with simulation predictions, validating the modelling. So as to refine the performance estimation, integration of additional contributions such as Von Karman turbulence profile, natural wind profile (neglected here), tiptilt vibrations and pointing residual jitter will be considered. Use of an end-to-end simulation tool shall also increase precision of the performance estimation.

These tests demonstrate however the ability of $\mathrm{AO}$ to correct the incident beam from turbulent effects and thus increase feasibility of single mode fiber injection. All these elements provide a solid ground to design efficient $\mathrm{AO}$ systems for satellite-to-ground laser links, with optimal 
injection into single mode fibers.. The next step will be to consider telescope diameters of several dozen centimeters, closer to those which are currently considered for satellite-to-ground laser links. Investigation on the influence of AO correction on data transmission still remains to be conducted experimentally. This will require the experimental setup to be upgraded with the addition of a single mode fiber and a high data rate digitization module. However, as the coherence time of power drops caused by AO residuals is significantly longer than the bit duration, the consequences on data transmission should be very comparable with those of smooth signal to noise ratio variations, at least for intensity based modulations like OOK. This conclusion could be generalized to phase modulations based techniques assuming the clock recovery of the coherent receiver is not affected by turbulence influence, which seems a rather common hypothesis ${ }^{24}$ although it still has to be demonstrated in the case of an $\mathrm{AO}$ corrected signal, the open literature on the subject being rather poor.

\section{Acknowledgments}

The author thanks the whole staff of Côte d'Azur Observatory for its kindness and availability during the experiments, Pr. A. Ziad and Dr. J. Chabé for kindly sharing GDIMM measurements of $\mathrm{r}_{0}$ during the pass.

\section{References}

1. R. K. Tyson et al., "Measurement of the bit-error rate of an adaptive optics, free-space laser communications system, part 2: multichannel configuration, aberration characterization, and closed-loop results", Opt. Eng. 44(9), 096003 (2005) [doi:10.1117/1.2043150].

2. T. Berkefeld et al., "Adaptive optics for satellite-to-ground laser communication at the $1 \mathrm{~m}$ Telescope of the ESA Optical Ground Station, Tenerife, Spain”, in Adaptive Optics Systems II, Proc. SPIE 7736, 77364C (2010) [doi:10.1117/12.857681]. 
3. K. E. Wilson, L. C. Roberts Jr., "Recent developments in adaptive optics for the lcrd optical ground station at table mountain," Proc. ICSOS 2014, Kobe, Japan (2014).

4. Wright, Malcolm W., et al. "Adaptive optics correction into single mode fiber for a low Earth orbiting space-to-ground optical communication link using the OPALS downlink." Optics Express 23.26 (2015): 33705-33712.

5. Fischer, E., et al. "Development, integration and test of a transportable adaptive optical ground station." 2015 IEEE International Conference on Space Optical Systems and Applications (ICSOS). IEEE, 2015.

6. N. Védrenne et al., "Turbulence effects on bi-directional ground-to-satellite laser communication systems”, Conference on Space Optical Systems and Applications, ICSOS, France (2012).

7. N. Védrenne et al., "Characterization of atmospheric turbulence for LEO to ground laser beam propagation at low elevation angles”, Proc. ICSOS 2014, Kobe, Japan (2014)

8. E. Samain, A. Abchiche, D. Albanese, et al., "MeO: The new French lunar laser station", In Proc. 16th Int. Laser Ranging Workshop, 2008.

9. M. Toyoshima et al., "Small Optical Transponder for Small Satellites", in Communication Systems Networks and Digital Signal Processing (CSNDSP), 2010 7th International Symposium on IEEE, 558-561, (2010)

10. cwe.ccsds.org/sls/docs/SLS-OPT/Meeting Materials/2014/Fall 2014 London meeting/Proposal of physical layer elements for a CCSDS OPT standard suitable for LEO to ground High Data Rate DTE link----.pdf

11. D.-H. Phung ; E. Samain ; N. Maurice, et al., "Telecom and scintillation first data analysis for DOMINO: laser communication between SOTA, onboard SOCRATES satellite, and MEO optical ground station", Proc. SPIE 9739, Free-Space Laser Communication and Atmospheric Propagation XXVIII, 973909 (March 15, 2016). 
12. C. Petit et al., "First laboratory validation of vibration filtering with LQG control law for Adaptive Optics", Opt. Exp. 16(1), 87-97 (2008)

13. D. P. Greenwood, "Bandwidth specification for adaptive optics systems," J. Opt. Soc. Am. 67, 390-393 (1977)

14. R. Noll, "Zernike polynomials and atmospheric turbulence”, JOSA 66(3), 207-211, 1976.

15. D. L. Fried, "Statistics of a Geometric Representation of Wavefront Distortion", JOSA 55 (11), 1427-1435, 1965.

16. E. Aristidi, Y. Fanteï-Caujolle, A. Ziad, C. Dimur, J. Chabé and B. Roland, “A new generalized differential image motion monitor", in Astronomical Telescopes+ Instrumentation, Proc. SPIE 9145, 91453G (2014) [doi:10.1117/12.2056201].

17. S. Gousset, “Optimisation d'un système d'Optique Adaptative pour l'observation de l'espace et mise en oeuvre d'un détecteur infra-rouge innovant en analyse de front d'onde", $\mathrm{PhD}$ thesis, Université Paris Diderot, (2015).

18. J.-M. Conan, G. Rousset, P.-Y. Madec, "Wave-front temporal spectra in high-resolution imaging through turbulence", JOSA A 12(7), 1559-1570 (1995).

19. C. Robert, J.-M. Conan, V. Michau, J. Renard, F. Dalaudier, "Retrieving parameters of the anisotropic refractive index fluctuations spectrum in the stratosphere from balloon-borne observations of stellar scintillation", JOSA A 25(2), 379-393 (2008).

20. C. Ruilier, and F. Cassaing, "Coupling of large telescopes and single-mode waveguides: application to stellar interferometry", JOSA A 18(1), 143-149 (2001)

21. S. Shaklan, F. Roddier, "Coupling starlight into single-mode fiber optics", Applied Optics 27 (11), 2334-2338 (1988)

22. N. Védrenne, J.-M. Conan, C. Petit, and V. Michau, "Adaptive optics for high data rate satelliteto-ground laser link", in Free-Space Laser Communication and Atmospheric Propagation XXVIII, Proc. SPIE 9739, 97390E (2016), [doi: 10.1117/12.2218213] 
23. Y. Koyama, T. Takayama, M. Akioka, H. Takenaka, Y. Munemasa, M. Toyoshima, "Completion of the small optical transponder for satellite-ground laser communication demonstration", in 2013 JOINT CONFERENCE of 19th Ka and Broadband communications and 31st AIAA International Communications Satellite Systems Conference, ICSSC, 17-2 (2013).

24. Belmonte, A., \& Kahn, J. M. (2010). "Efficiency of complex modulation methods in coherent free-space optical links," Optics express, 18 (4), 3928-3937.

Cyril Petit is a senior research scientist at Onera (France) with 10 year experience in Adaptive Optics (AO). He graduated from ENSTA, then obtained his Ph.D. in 2006. He is involved in various research activities in $\mathrm{AO}$ applied to astronomy, space observation, biomedical imaging and free space optical telecommunication. His research interests include design, numerical simulation, experimental implementation and testing of AO systems, with a focus on control. He was in charge of SPHERE AO control design and AIT.

\section{Caption List}

Fig. 1 Picture of $\mathrm{MeO}$ telescope.

Fig. 2 Illustration of SOCRATES (credit AES) (left) and picture of SOTA (credit NICT, NEC) (right).

Fig. 3 Schematic of the ODISSE AO bench at MeO Coudé focus

Fig. 4 Schematic of the DM actuator grid (dots), located at the angles of SH-WFS subaperture grid (squares). The useful aperture, with telescope spider and central occultation are delimited in red. A 8x8 sub aperture grid is used on SH-WFS, valid subapertures are whitened. 
Fig. 5 Top: elevation as a function of time since the rise of the satellite. Middle: distance to satellite, bottom: antenna slew rate. Blue: WFS data acquisition.

Fig. 6 Wavefront variance per Zernike polynomial radial order for the five selected elevations experimental (squares), compared to theory (- - -).

Fig. 7 Fried parameter evaluation as a function of elevation, during the pass (lines) and on a star (symbols) after the pass. Solid line corresponds to WFS measurements, each value is provided with a one sigma error bar. Dashed line corresponds to evolution as deduced from DIMM measurement and Eq. (1). Dashed dotted red line is the same as previous line with introduction of bias. Grey vertical band indicates period of time dedicated to parameters adjustment and loop closure, leading to hampered evaluation. The results of estimation from WFS data acquired on a star (Enif) at 22:53:25 is plotted in black diamonds. Simultaneous GDIMM measurement is plotted with the black cross, red triangle corresponds to the DIMM measurements with the bias.

Fig.8 Temporal spectra for radial orders from 2 to 6 for the $32.6^{\circ}$ elevation. Cutoff frequencies for each radial orders are reported (vertical mixed color lines).

Fig.9 Temporal spectra for radial order 6 for the $32.6^{\circ}$ elevation. Asymptotes enabling the identification of the three main layers are reported. Green: loaw altitude layer, red: intermediate layer, blue: high altitude layer. Vertical mixed color lines: frequencies corresponding to each layer.

Fig. 10 Custom $\mathrm{C}_{\mathrm{n}}^{2}$ (Multi-Gaussian) and Hufnagel - Valley profile.

Fig. 11 Flux fluctuations power spectral densities for $28.2^{\circ}, 32.6^{\circ}$ and 41.9 .

Fig. 12 Results of analytical model compared to temporal PSD for radial orders 2 to 6 for $32.6^{\circ}$ elevation. 
Fig. 13 Evolution of temporal PSD of sixth radial order for increasing elevation.

Fig. 14 Longitudinal extension of the stationarity zone of the turbulence layer located at altitude $h$.

Fig. 15 Evolution of the scintillation index as a function of elevation from measurements (black), and from multi-gaussian profile (dashed red).

Fig. 16 Long exposure images (linear scale) obtained in open (left) or closed AO loop (right), with same scaling. Open-loop image is hardly visible, due to a factor 45 in maximum intensity and $\sim 7$ in FWHM.

Fig. 17 Short exposure images (linear scale) obtained in open (left) or closed AO loop (right). For better visualisation, both images have been normalized to their maximum. On right image a zoom has been proposed to represent (white circle) the equivalent fiber Gaussian mode intensity with $\omega$.

Fig. 18 Time evolution of PIB along frame number, first in open then in closed loop (dashed line delimits the separation).

Fig. 19 Time evolution of PIB along frame number, compared to coupling efficiency, both obtained in numerical simulation.

Fig. 20 Compared temporal PSD of PIB as obtained experimentally (at $100 \mathrm{~Hz}$ sampling frequency) in red or by numerical simulation. A good matching is obtained in the accessible frequency range.

Fig. 21 Left: compared statistics (probability density) of PIB as obtained experimentally (red) or by numerical simulation (black). CE obtained in simulation with similar time averaging (10 ms) is added in blue. Right: statistics of PIB (black) and CE (blue) as obtained in numerical 
simulation for full frame rate $(1.45 \mathrm{kHz})$ (dashed line) or simulated $10 \mathrm{~ms}$ time averaging (exposure time) (solid line).

Fig. 22 AO loop performance in terms of wavefront variance $\left(\mathrm{rad}^{2}\right)$ as reconstructed from closed-loop slopes (Experimental, in red) and as estimated from the simulation (in black), at laser wavelength. Vertical dotted lines correspond to time interval for PIB estimation from PCO images.

Fig. 23 Temporal evolution of tip mode in open loop. A clear drift is visible due to telescope residual pointing error.

Fig. 24 Cumulated temporal Power Spectrum Density of tip mode in closed loop 